\title{
A global theory of flexes of periodic functions
}

\author{
by Gudlaugur Thorbergsson and Masaaki Umehara
}

\begin{abstract}
For a real valued periodic smooth function $u$ on $\mathbf{R}, n \geq 0$, one defines the osculating polynomial $\varphi_{s}$ (of order $2 n+1$ ) at a point $s \in \mathbf{R}$ to be the unique trigonometric polynomial of degree $n$, whose value and first $2 n$ derivatives at $s$ coincide with those of $u$ at $s$. We will say that a point $s$ is a clean maximal flex (resp. clean minimal flex) of the function $u$ on $S^{1}$ if and only if $\varphi_{s} \geq u$ (resp. $\varphi_{s} \leq u$ ) and the preimage $(\varphi-u)^{-1}(0)$ is connected. We prove that any smooth periodic function $u$ has at least $n+1$ clean maximal flexes of order $2 n+1$ and at least $n+1$ clean minimal flexes of order $2 n+1$. The assertion is clearly reminiscent of Morse theory and generalizes the classical four vertex theorem for convex plane curves.
\end{abstract}

\section{$\S 1$ Introduction}

For a real valued $C^{2 n}$-function $u$ on $S^{1}=\mathbf{R} / 2 \pi \mathbf{Z}, n \geq 0$, one defines the osculating polynomial $\varphi_{s}$ (of order $\left.2 n+1\right)$ at a point $s \in S^{1}$ to be the unique trigonometric polynomial of degree $n$,

$$
\varphi_{s}(t)=a_{0}+a_{1} \cos t+b_{1} \sin t+\cdots+a_{n} \cos n t+b_{n} \sin n t
$$

whose value and first $2 n$ derivatives at $s$ coincide with those of $u$ at $s$. If $u$ is $C^{2 n+1}$ and the value and the first $2 n+1$ derivatives of $u$ and $\varphi_{s}$ coincide in $s$, i.e., if $\varphi_{s}$ hyperosculates $u$ in $s$, then we call $s$ a flex of $u$ (of order $2 n+1)$. Notice that the order $2 n+1$ of the osculating polynomials and flexes in the definition above has been chosen such that it coincides with the dimension of the space $A_{2 n+1}$ of trigonometric polynomials of degree $n$. Notice also that a flex of order one, i.e. the case $n=0$, is nothing but a critical point. The existence of $2 n+2$ flexes of order $2 n+1$ for any $C^{2 n+1}$-function $u$ on $S^{1}$ is an easy consequence of the well-known fact that a function has at least $2 n+2$ zeros if its Fourier coefficients $a_{i}$ and $b_{i}$ vanish for $i \leq n$; see Appendix A for a proof. Here we will prove the much more difficult result that there are $2 n+2$ such flexes satisfying the global property that the osculating polynomials $\varphi_{s}$ in the flexes support $u$, i.e., either $\varphi_{s} \leq u$ or $u \leq \varphi_{s}$. More precisely, we will say that a point $s$ is a clean maximal flex (resp. clean minimal flex) of a $C^{2 n}$-function $u$ on $S^{1}$ if and only if $\varphi_{s} \geq u$ (resp. $\varphi_{s} \leq u$ ) and the preimage $(\varphi-u)^{-1}(0)$ is connected. This terminology is compatible with our definition of a flex, since it is easy to see that a clean maximal (or minimal) flex is a flex if $u$ is $C^{2 n+1}$.

Our main result is the following theorem.

Mathematics Subject Classification (2000). Primary 51L15 Secondary 53C75, 53A15 
1.1 Theorem. Let $u$ be a real valued $C^{2 n}$-function on $S^{1}$ where $n \geq 1$. Then $u$ has at least $n+1$ clean maximal flexes of order $2 n+1$ and at least $n+1$ clean minimal flexes of order $2 n+1$.

The theorem is not true if $n=0$. A continuous function $u$ on $S^{1}$ is obviously supported by constant functions in points where $u$ takes on its maximum and minimum values, but it does not have to be true that $u$ takes on its maximum and minimum value in connected sets.

The above theorem is clearly reminiscent of Morse theory. We would like to point out a further similarity. Assume that $u$ is a $C^{2}$-function on $S^{1}$ and define the function $\varphi_{s}^{\bullet}$ for every $s \in S^{1}$ as the largest function in $A_{3}$ such that $\varphi_{s}^{\bullet} \leq u$ and $\varphi_{s}^{\bullet}(s)=u(s)$. Typically, $u$ and $\varphi_{s}^{\bullet}$ have two common values. A point $s$ in $S^{1}$ is therefore exceptional if $u$ and $\varphi_{s}^{\bullet}$ have only a common value in $s$ or if $u$ and $\varphi_{s}^{\bullet}$ have more than two common values. In the first exceptional case we have that $s$ is a minimal flex. We denote the number of such flexes (or the corresponding functions $\varphi_{s}^{\bullet}$ ) by $s^{\bullet}$. Let $t^{\bullet}$ denote the number of functions $\varphi_{s}^{\bullet}$ counted with multiplicities having more than two values in common with $u$. (If $\varphi_{s}^{\bullet}$ and $u$ have $k$ values in common, the $\varphi_{s}^{\bullet}$ contributes $k-2$ to the number $t^{\bullet}$.) If $s^{\bullet}$ is finite, then $t^{\bullet}$ is finite too and the following formula holds:

$$
s^{\bullet}-t^{\bullet}=2 .
$$

A similar formula holds for the functions $\varphi_{s}^{\circ}$ defined for every $s \in S^{1}$ as the smallest function in $A_{3}$ such that $\varphi_{s}^{\circ} \geq u$ and $\varphi_{s}^{\circ}(s)=u(s)$. The two formulas taken together generalize Theorem 1.1 for $n=1$. One should expect such formulas to hold for every $n$, thus giving a far-reaching generalization of Theorem 1.1, but so far there is no such result. The above formula implies a theorem on strictly convex curves that was first proved by Bose [Bo] in the generic case and then generalized by Haupt [Ha] to generic simple closed curves. It was proved for general simple closed curves by the second author [Um] using intrinsic circle systems, a method that will be generalized in the present paper.

If $n=1$, the existence of four flexes of order three on a periodic function does in fact imply the so-called four vertex theorem for strictly convex curves in the Euclidean plane. A smooth regular curve $\gamma$ has at any point $s$ an osculating circle which can be defined as the unique circle having at least a three point contact with $\gamma$ in $s$. The point $s$ is called a vertex of $\gamma$ if the osculating circle at $s$ has at least a four point contact with $\gamma$ in $s$, or, in other words, the osculating circle hyperosculates $\gamma$ in $s$. The four vertex theorem for strictly convex curves says that such curves have at least four vertices. Theorem 1.1 now implies that there are at least two vertices at which the osculating circles are inscribed and at least two vertices at which they are circumscribed. This result is more generally true for any simple closed curve in the Euclidean plane, see [Kn], and also follows from the methods we use here, see [Um], but in this generality the curves do not correspond to functions on $S^{1}$.

We now describe the connection between strictly convex curves and periodic functions in more detail. Fix a point $o$ in the interior of a strictly convex curve $\gamma$ in the $(x, y)$-plane. 


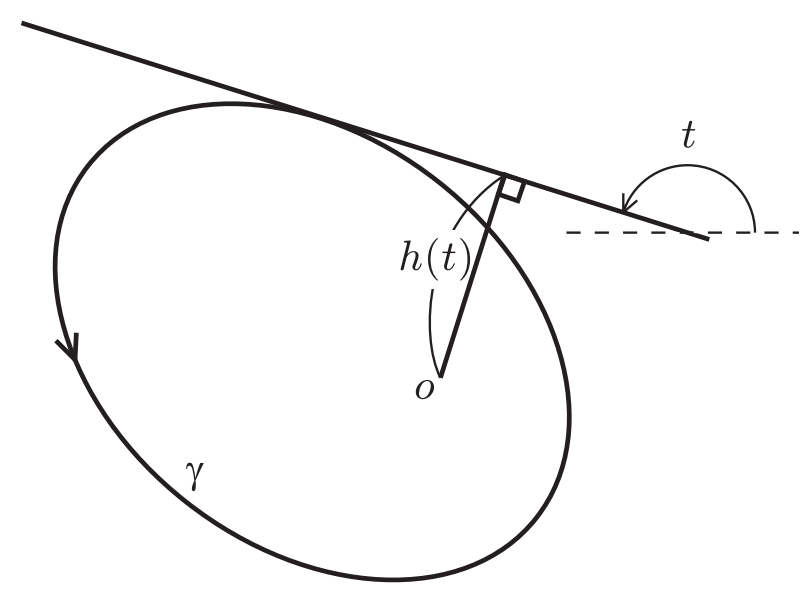

Figure 1.

For each $t \in[0,2 \pi)$, there is a unique tangent line $L(t)$ of the curve which makes angle $t$ with the $x$-axis. Let $h(t)$ be the distance between $o$ and the line $L(t)$. The function $h$ is called the supporting function of the curve $\gamma$ with respect to o. The radius of the osculating circle of $\gamma$ at $t$ is given by $h^{\prime \prime}(t)+h(t)$. It can easily be checked that a point $t$ is a vertex of the curve if and only if $h^{\prime \prime \prime}(t)+h^{\prime}(t)$ vanishes. Let $\varphi_{s}$ be the osculating function (of order three) of $h$ in $s$. Then, by definition, $h(s)=\varphi_{s}(s), h^{\prime}(s)=\varphi_{s}^{\prime}(s)$ and $h^{\prime \prime}(s)=\varphi_{s}^{\prime \prime}(s)$. Furthermore, $s$ is a flex if and only if $h^{\prime \prime \prime}(s)=\varphi_{s}^{\prime \prime \prime}(s)$. Notice that $\varphi_{s}$ is a trigonometric polynomial of degree one, i.e.,

$$
\varphi_{s}(t)=a_{0}+a_{1} \cos t+b_{1} \sin t .
$$

The function $\varphi_{s}^{\prime \prime \prime}+\varphi_{s}^{\prime}$ clearly vanishes identically. Hence $s$ is a flex of $h$ if and only if $h^{\prime \prime \prime}(s)+h^{\prime}(s)=0$. Hence we see that there is a one-to-one correspondence between the vertices of the curve $\gamma$ and the flexes of the function $h$. It is also easy to see that a clean maximal (resp. minimal) flex of $h$ corresponds to a vertex where the osculating circle is inscribed (resp. circumscribed) and touches $\gamma$ in a connected set.

The methods of the paper are general and can be applied in other situations. Let $\gamma$ again denote a strictly convex closed curve that we assume to be contained in the affine plane (or in the projective plane, but this is not more general since there is always a line which such a curve does not meet). One defines the osculating conic $C_{t}$ at a point $t$ of $\gamma$ to be the unique conic which meets $\gamma$ with multiplicity at least five in $t$. If $C_{t}$ and $\gamma$ meet with multiplicity at least six in $t$, then $t$ is called a sextactic point. If the osculating conic at $t$ is inscribed (resp. circumscribed) and meets $\gamma$ in a connected set, then we will call $t$ a clean maximal (resp. clean minimal) sextactic point. One can show that clean maximal and clean minimal sextactic points are in fact sextactic. We will prove the following theorem that improves a result of the authors in [TU2] as well as a result of Mukhopadhyaya in [Mu2].

1.2 Theorem. A strictly convex curve has at least three clean maximal sextactic points and three clean minimal sextactic points.

The singularities we have been discussing so far are flexes of periodic functions and vertices and sextactic points of convex curves. In all three cases the dimension of the space of approximating functions or curves is odd. (The space of circles is three-dimensional and 
the space of conics is five-dimensional.) There are also singularities of even order of which inflection points of curves in the projective plane are the most important example. Here one approximates the curve with lines which form a two-dimensional space. In the even order case one typically has to deal with nonorientable situations like noncontractible curves in the projective plane or antisymmetric functions. In Appendix A we also deal with even order flexes of antisymmetric functions, but we restrict ourselves to the odd order case in the main body of the paper since the even order case is considerably more difficult due to problems with nonorientability.

In Section two we introduce our main tool, the intrinsic systems, which are in our applications analogues of intersection divisors on algebraic curves. This approach is a generalization of the methods in [Um], [TU1], [TU2], and the idea behind it is inspired by the paper $[\mathrm{Kn}]$ of Kneser. The situation we deal with in Section two and in the rest of the paper is somewhat more general than in the introduction since we treat osculating functions that do not need to be trigonometric functions. In Section three we start drawing consequences from the defining axioms of an intrinsic system. In Section four we generalize a result of Jackson [Ja]. In Section five we prove Theorem 1.1 and Theorem 1.2. Section six contains a remark and some questions on the possible arrangement of the clean maximal and minimal flexes whose existence was proved in Section five. In Appendix A some basic properties of trigonometric polynomials are explained in the more general setting of Chebyshev spaces. In Appendix B we explain an elementary analytic result that is used in the paper.

\section{$\S 2$ Chebyshev spaces and intrinsic systems of periodic functions}

A real valued continuous function $u$ on $S^{1}$ is said to be piecewise $C^{2 n}$ if it is of class $C^{2 n}$ except at finitely many points $s_{1}, \ldots, s_{m}$, and if, furthermore, $\left.u\right|_{\left[s_{i}, s_{i+1}\right]}$ can be extended to a $C^{2 n}$-function on an open interval containing $\left[s_{i}, s_{i+1}\right]$ for all $i=1, \ldots, m$, where we understand $m+1$ to mean 1 . We will refer to $s_{1}, \ldots, s_{m}$ as singular points or singularities of $u$.

Our goal is to study the existence of clean flexes of order $2 n+1$ of a $C^{2 n}$-function $u$ on $S^{1}$ that does not have any singularities. In the proofs below, we will frequently have to modify the function $u$ by restricting it to an interval and then extending it to the complement of the interval by piecewise trigonometric polynomials. So we shall frequently have to deal with piecewise $C^{2 n}$-periodic functions.

We let $A_{2 n+1}$ denote the vector space of trigonometric polynomials of degree at most $n$, i.e.,

$$
A_{2 n+1}=\left\{\varphi(t)=a_{0}+\sum_{k=1}^{n}\left(a_{k} \cos k t+b_{k} \sin k t\right)\right\} .
$$

The space $A_{2 n+1}$ is an example of a Chebyshev space of order $2 n+1$; see Appendix A where this concept is introduced and discussed in detail. We repeat here the definition of Chebyshev spaces of odd order.

2.1 Definition. A linear subspace $\mathcal{A}$ of $C^{2 n}(\mathbf{R} / 2 \pi)$ is called a Chebyshev space of order $2 n+1$ if its dimension is at least $2 n+1$ and if the number of zeros in $[0,2 \pi)$, counted with multiplicities, of a nonvanishing function in $\mathcal{A}$ is at most $2 n$. 
It will be proved in Appendix A that the dimension of a Chebyshev space is always equal to its order. Let $u: S^{1} \rightarrow \mathbf{R}$ be a $C^{2 n}$-function. Then for each $s \in S^{1}$ there exists a unique function $\varphi_{s} \in \mathcal{A}$ whose value and first $2 n$ derivatives at $s$ coincide with those of $u$ in $s$. We refer to Theorem A.2 in Appendix A for a proof of the existence of $\varphi_{s}$. We call $\varphi_{s}$ the $\mathcal{A}$-osculating function of $u$ at $s$. If both $u$ and $\varphi_{s}$ are $C^{2 n+1}$-functions and the value and the first $2 n+1$ derivatives of $u$ and $\varphi_{s}$ coincide in $s$, then we call $s$ an $\mathcal{A}$-flex of $u$.

We will from now on work with an arbitrary Chebyshev space $\mathcal{A}$ of order $2 n+1$. The reader may want to think of $\mathcal{A}$ as simply being $A_{2 n+1}$. Notice though that the more general point of view is quite useful even when one is primarily interested in $A_{2 n+1}$. For an example of this, see the space $\mathcal{A}_{\psi_{1}}$ that is used to prove Theorem 1.1 from the introduction in Section five.

Throughout the paper we let $I$ either denote the whole $S^{1}$ or a nonempty proper closed interval $[a, b]$ on $S^{1}$. In both cases we will refer to $I$ as an interval.

2.2 Definition. Let $u$ be a piecewise $C^{2 n}$-function. Let $I=[a, b]$ be a proper closed interval on $S^{1}$ and $\left(\iota_{a}, \iota_{b}\right)$ a pair of nonnegative integers which are less than or equal to $\infty$. Then $u$ is said to satisfy the boundary regularity condition $\left(\iota_{a}, \iota_{b}\right)$ on $I$,

(1) if $u$ is at least $C^{2 \iota_{a}-1}$ in $a$, but not $C^{2 \iota_{a}+1}$, and at least $C^{2 \iota_{b}-1}$ in $b$, but not $C^{2 \iota_{b}+1}$,

(2) and if $u$ is not $C^{2 \iota_{a}}$ in $a$ (resp. not $C^{2 \iota_{b}}$ in $b$ ), then the $2 \iota_{a}$-th (resp. $2 \iota_{b}$-th) derivative of $u$ from the left at $a$ (resp. right at $b$ ) is greater than that from the right at $a$ (resp. left at $b$ ).

Let $I$ be a proper closed interval. We let $I_{\left(\iota_{a}, \iota_{b}\right)}^{n}$ denote the subset of the Cartesian product $I^{n}$ consisting of those elements $\left(p_{1}, \ldots, p_{n}\right)$ of $I^{n}$ with at most $\iota_{a}$ components equal to the endpoint $a$ and at most $\iota_{b}$ components equal to the endpoint $b$. For example,

$$
\begin{aligned}
& I_{(0,0)}^{2}=(a, b) \times(a, b), \\
& I_{(1,0)}^{2}=\{(x, y) \in[a, b] \times[a, b] ;(x, y) \neq(a, a), x \neq b, y \neq b\}, \\
& I_{(1,1)}^{2}=\{(x, y) \in[a, b] \times[a, b] ;(x, y) \neq(a, a),(b, b)\}, \\
& I_{(2,1)}^{2}=\{(x, y) \in[a, b] \times[a, b] ;(x, y) \neq(b, b)\}, \\
& I_{(2,2)}^{2}=[a, b] \times[a, b] .
\end{aligned}
$$

We next prove the following lemma.

2.3 Lemma. Let $u$ be a piecewise $C^{2 n}$-function on $S^{1}$ and let $I$ be a nonempty closed interval of $S^{1}$ that is either proper or the whole circle. We suppose that $u$ is $C^{2 n}$ on $I$ and satisfies the boundary regularity condition $\left(\iota_{a}, \iota_{b}\right)$ if $I$ is a proper interval. If $I=S^{1}$ we assume that $u$ is $C^{2 n}$ on the whole $S^{1}$. For $\left(p_{1}, \ldots, p_{n}\right) \in I_{\left(\iota_{a}, \iota_{b}\right)}^{n}\left(\right.$ or $\left(p_{1}, \ldots, p_{n}\right) \in I^{n}$ if $\left.I=S^{1}\right)$ let $\Lambda$ denote the one-dimensional affine space of functions $\varphi \in \mathcal{A}$ such that

$$
\varphi^{(k)}\left(p_{i}\right)=u^{(k)}\left(p_{i}\right) \quad \text { for all } \quad k=0, \ldots, 2 \mu_{i}-1 \text { and all } i=1, \ldots, n,
$$

where $\mu_{i}$ is the number of components of $\left(p_{1}, \ldots, p_{n}\right)$ equal to $p_{i}$. Then the subset of functions $\varphi \in \Lambda$ such that $\varphi \geq u$ is a nonempty closed interval that we denote by $\Lambda_{u}\left(p_{1}, \ldots, p_{n}\right)$. 
Proof. By definition, $\varphi \in \Lambda$ if and only if

$$
\varphi^{(k)}\left(p_{i}\right)=u^{(k)}\left(p_{i}\right) \quad \text { for all } \quad k=0, \ldots, 2 \mu_{i}-1 \text { and all } i=1, \ldots, n .
$$

It follows from Theorem A.2 in Appendix A that $\Lambda$ is a one-dimensional affine subspace of $\mathcal{A}$. Let $\varphi_{1}$ be an arbitrary function in $\Lambda$. Take another function $\varphi_{2} \in \mathcal{A}$ satisfying

$$
\varphi_{2}^{(k)}\left(p_{i}\right)=0 \quad \text { for all } \quad k=0, \ldots, 2 \mu_{i}-1 \text { and all } i=1, \ldots, n
$$

which is not identically zero. Then $\varphi_{2}^{\left(2 \mu_{i}\right)}\left(p_{i}\right) \neq 0$ for all $i=1, \ldots, n$ (cf. Definition 2.1). Notice that $\varphi_{2}$ cannot change sign in any of the points $p_{i}$ since its first nonvanishing derivative there is of an even order. Since the function $\varphi_{2}$ has at most $2 n$ zeros counted with multiplicities, either $\varphi_{2} \geq 0$ or $\varphi_{2} \leq 0$ holds. So we may assume $\varphi_{2} \geq 0$. Then $\varphi_{2}^{\left(2 \mu_{i}\right)}\left(p_{i}\right)>0$ for all $i=1, \ldots, n$. For every natural number $m \in \mathbf{N}$, we define a function $v_{m}$ on $S^{1}$ by setting

$$
v_{m}(t)=-u(t)+\varphi_{1}(t)+m \varphi_{2}(t) .
$$

There is an $m_{0} \in \mathbf{N}$ such that for all $m \geq m_{0}$ we have that $v_{m}$ and its first $2 \mu_{i}-1$ derivatives vanish in $p_{i}$, but $v_{m}^{\left(2 p_{i}\right)}\left(p_{i}\right)>0$, for all $i=1, \ldots, n$, except when $p_{i}$ is either $a$ or $b$ where $u$ might only be $C^{2 \iota_{a}-1}$ or $C^{2 \iota_{b}-1}$ respectively. In case $p_{i}$ is $a$ (resp. $b$ ) and $u$ is only $C^{2 \mu_{i}-1}$, i.e., $\mu_{i}=\iota_{a}$ (resp. $\mu_{i}=\iota_{b}$ ), we choose $m_{0}$ sufficiently large so that the $2 \mu_{i}$-th derivative from the left and from the right of $v_{m}$ are both positive in $p_{i}$. Hence there is a neighborhood of $\left\{p_{1}, \ldots, p_{n}\right\}$ on which $v_{m}$ is nonnegative. On the complement of this neighborhood, we have that $\varphi_{2}$ is bounded from below by a positive number. Hence there is a $m_{1} \geq m_{0}$ such that $v_{m} \geq 0$ for $m \geq m_{1}$. Therefore the function

$$
\varphi(t)=\varphi_{1}(t)+m \varphi_{2}(t)
$$

is in $\Lambda$ and satisfies $\varphi \geq u$. The rest of the lemma is now clear.

Let $u$ be a function as in Lemma 2.3. Now we can begin to associate what will call an intrinsic system to the function $u$. This is easy if $I$ is equal to the whole circle $S^{1}$ and the definition consists only of the two cases (i), (ii) and (iv) below. We will therefore restrict ourselves to the more difficult case of functions that are $C^{2 n}$ on a proper closed interval $I$ satisfying a boundary regularity condition $\left(\iota_{a}, \iota_{b}\right)$. We have seen in Lemma 2.3 that the subset $\Lambda_{u}\left(p_{1}, \ldots, p_{n}\right)$ of $\mathcal{A}$ is a nonempty closed interval for each $\left(p_{1}, \ldots, p_{n}\right) \in I_{\left(\iota_{a}, \iota_{b}\right)}^{n}$. We define the function $\varphi_{\left(p_{1}, \ldots, p_{n}\right)} \in \Lambda_{u}\left(p_{1}, \ldots, p_{n}\right)$ to be the boundary point of this interval, or, what is the same thing, as

$$
\varphi_{\left(p_{1}, \ldots, p_{n}\right)}=\inf \left\{\psi \in \Lambda_{u}\left(p_{1}, \ldots, p_{n}\right)\right\} .
$$

We call $\varphi_{\left(p_{1}, \ldots, p_{n}\right)}$ the minimal function of $u$ with respect to $\left(p_{1}, \ldots, p_{n}\right)$.

We denote by $\mathbf{N}_{0}$ the set of nonnegative integers, and denote by $\operatorname{Map}\left(S^{1}, 2 \mathbf{N}_{0} \cup\{\infty\}\right)$ the set of maps from $S^{1}$ to $2 \mathbf{N}_{0} \cup\{\infty\}$. We define a map

$$
f_{u}: I_{\left(\iota_{a}, \iota_{b}\right)}^{n} \rightarrow \operatorname{Map}\left(S^{1}, 2 \mathbf{N}_{0} \cup\{\infty\}\right),
$$


by setting

$$
f_{u}\left(p_{1}, \ldots, p_{n}\right)(q)=0
$$

for any $q \in S^{1}$ such that $u(q) \neq \varphi_{\left(p_{1}, \ldots, p_{n}\right)}(q)$;

$$
f_{u}\left(p_{1}, \ldots, p_{n}\right)(q)=2 k
$$

if $q \in I^{\circ}$ (where $I^{\circ}$ denotes the interior of $I$ ), $u(q)=\varphi_{\left(p_{1}, \ldots, p_{n}\right)}(q)$ and precisely $2 k-1$ derivatives of $u$ and $\varphi_{\left(p_{1}, \ldots, p_{n}\right)}$ agree in $q$ and $k \leq n$;

$$
f_{u}\left(p_{1}, \ldots, p_{n}\right)(q)=2 k
$$

if $q=a$ (resp. $b), u(q)=\varphi_{\left(p_{1}, \ldots, p_{n}\right)}(q)$ and the first $2 k-1$ derivatives of $u$ and $\varphi_{\left(p_{1}, \ldots, p_{n}\right)}$ agree in $q$, the $2 k$-th derivative of $\varphi_{\left(p_{1}, \ldots, p_{n}\right)}$ is different from the $2 k$-th derivative of $u$ from the right in $a$ (resp. the left in $b)$ and $k \leq \iota_{a} \leq n$ (resp. $\left.\leq \iota_{b} \leq n\right)$;

$$
f_{u}\left(p_{1}, \ldots, p_{n}\right)(q)=\infty
$$

if $q \in I^{\circ}, u(q)=\varphi_{\left(p_{1}, \ldots, p_{n}\right)}(q)$ and more than $2 n-1$ derivatives of $u$ and $\varphi_{\left(p_{1}, \ldots, p_{n}\right)}$ agree in $q$;

$$
f_{u}\left(p_{1}, \ldots, p_{n}\right)(q)=\infty
$$

if $q=a$ (resp. $q=b$ ) and $u(t)$ is $C^{2 n}$ at $q, u(q)=\varphi_{\left(p_{1}, \ldots, p_{n}\right)}(q)$ and more than $2 n-1$ derivatives of $u$ and $\varphi_{\left(p_{1}, \ldots, p_{n}\right)}$ agree in $q$;

$$
f_{u}\left(p_{1}, \ldots, p_{n}\right)(q)=2
$$

if $q \notin I$ and $u(q)=\varphi\left(p_{1}, \ldots, p_{n}\right)(q)$.

This ends the definition of the map

$$
f_{u}: I_{\left(\iota_{a}, \iota_{b}\right)}^{n} \rightarrow \operatorname{Map}\left(S^{1}, 2 \mathbf{N}_{0} \cup\{\infty\}\right)
$$

It will frequently be convenient to use the following notation:

$$
\begin{gathered}
f_{u}\left(p^{k}, p_{k+1}, \ldots, p_{n}\right)=f_{u}\left(p, \ldots, p, p_{k+1}, \ldots, p_{n}\right), \\
f_{u}\left(p^{k}, q^{l}, p_{k+l+1}, \ldots, p_{n}\right)=f_{u}\left(p, \ldots, p, q, \ldots, q, p_{k+l+1}, \ldots, p_{n}\right),
\end{gathered}
$$

and so on. We will denote the support of $f_{u}\left(p_{1}, \ldots, p_{n}\right)$ by $F_{u}\left(p_{1}, \ldots, p_{n}\right)$, i.e.,

$$
F_{u}\left(p_{1}, \ldots, p_{n}\right)=\left\{r \in S^{1} \mid f_{u}\left(p_{1}, \ldots, p_{n}\right)(r)>0\right\}
$$

The value of $f_{u}\left(p_{1}, \ldots, p_{n}\right)$ at a point $r$ will be called the multiplicity of $r$ with respect to $f_{u}\left(p_{1}, \ldots, p_{n}\right)$. The sum over all values of $f_{u}\left(p_{1}, \ldots, p_{n}\right)$, which can of course be infinite, will be called the total multiplicity of $f_{u}\left(p_{1}, \ldots, p_{n}\right)$. 
A point $s$ in $S^{1}$ will be called a global $\mathcal{A}$-flex of $u$ if its multiplicity with respect to $f_{u}\left(s^{n}\right)$ is $\infty$. Notice that a point $s \in I$ is a a global $\mathcal{A}$-flex if and only if $\varphi_{\left(s^{n}\right)}$ is defined and equal to the $\mathcal{A}$-osculating function $\varphi_{s}$ of $u$ at $s$. In particular, a global $\mathcal{A}$-flex is an $\mathcal{A}$-flex when $u$ and $\varphi_{s}$ are both $C^{2 n+1}$. However, the converse is not true. In fact, it is clear that a global $\mathcal{A}$-flex $s \in I$ has the global property that the osculating function $\varphi_{s}$ of $u$ at $s$ is greater than or equal to $u$ over the whole circle $S^{1}$. A global $\mathcal{A}$-flex $s$ is called a clean maximal flex if the preimage $\left(\varphi_{s}-u\right)^{-1}(0)$ is connected. If $u$ is $C^{2 n}$ on $S^{1}$, then we can also define the intrinsic system $f_{(-u)}$. A clean maximal $\mathcal{A}$-flex of $-u$ is called a clean minimal flex. Phrased differently, a point $s$ is a clean maximal (resp. minimal) $\mathcal{A}$-flex of $u$ if and only if the osculating function $\varphi_{s}$ is greater (resp. less) than or equal to $u$ and the preimage $\left(\varphi_{s}-u\right)^{-1}(0)$ is connected.

Example. We give here an example which shows the difference between $\mathcal{A}$-flexes, global $\mathcal{A}$-flexes and clean $\mathcal{A}$-flexes when $\mathcal{A}=A_{2 n+1}$. Consider a $2 \pi$-periodic smooth function $u(t)$ satisfying $0 \leq u(t) \leq 1$ which is identically 1 on the closed interval $I=[2 \pi / 5,3 \pi / 5]$ and identically zero on the intervals $[0, \pi / 5]$ and $[4 \pi / 5,2 \pi]$.

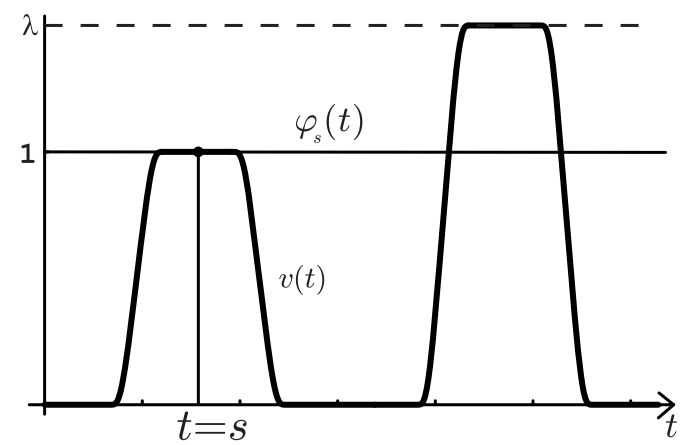

A flex which is not clean

Figure 2.

Next we set

$$
v(t)=u(t)+\lambda u(t+\pi) \quad \text { for } \quad \lambda \geq 0 .
$$

When $\lambda<1$, the points on the interval $I$ are clean maximal $A_{2 n+1}$-flexes of $v(t)$. If $\lambda=1$, the points on the interval $I$ are global $A_{2 n+1}$-flexes but not clean maximal $A_{2 n+1}$-flexes. Finally, if $\lambda>1$, the points on the interval $I$ are $A_{2 n+1}$-flexes, but not global $A_{2 n+1}$-flexes.

In the next proposition we bring the most basic properties of the map $f_{u}$ that we have associated to the function $u$. These properties will lead us to the notion of an intrinsic system, see Definition 2.5 below. Notice that there are two cases. The interval $I$ is either a proper closed interval of $S^{1}$ on which $u$ is $C^{2 n}$ and satisfies the boundary condition $\left(\iota_{a}, \iota_{b}\right)$ or $I$ is the whole circle $S^{1}$ and $u$ is $C^{2 n}$ on $S^{1}$. We will formulate the following proposition for the first case, i.e., for $f_{u}: I_{\left(\iota_{a}, \iota_{b}\right)}^{n} \rightarrow \operatorname{Map}\left(S^{1}, 2 \mathbf{N}_{0} \cup\{\infty\}\right)$, but notice that everything is equally true for $I=S^{1}$; one simply has to delete the index $\left(\iota_{a}, \iota_{b}\right)$ from $I_{\left(\iota_{a}, \iota_{b}\right)}^{n}$ and disregard (A.8).

2.4 Proposition. The map $f=f_{u}$ satisfies the following properties:

(A1) (Closedness) The set $F\left(p_{1}, \ldots, p_{n}\right)$ is closed for all $\left(p_{1}, \ldots, p_{n}\right) \in I_{\left(\iota_{a}, \iota_{b}\right)}^{n}$. 
(A2) (Symmetry) The functions $f\left(p_{1}, \ldots, p_{n}\right)$ and $f\left(p_{\sigma(1)}, \ldots, p_{\sigma(n)}\right)$ coincide for every permutation $\sigma \in \mathcal{S}_{n}$ and all $\left(p_{1}, \ldots, p_{n}\right) \in I_{\left(\iota_{a}, \iota_{b}\right)}^{n}$.

(A3) (Supporting Property) The support $F\left(p_{1}, \ldots, p_{n}\right)$ contains $\left\{p_{1}, \ldots, p_{n}\right\}$ for all $\left(p_{1}, \ldots, p_{n}\right) \in I_{\left(\iota_{a}, \iota_{b}\right)}^{n}$.

(A4) (Exchangeability) If $f\left(p_{1}, \ldots, p_{n}\right)(r) \geq 2 j$ for a point $r \in I$ that is different from $p_{1}, \ldots, p_{n-j}$ where $j \leq n$, then $\left(p_{1}, \ldots, p_{n-j}, r^{j}\right) \in I_{\left(\iota_{a}, \iota_{b}\right)}^{n}$ and $f\left(p_{1}, \ldots, p_{n-j}, r^{j}\right)=$ $f\left(p_{1}, \ldots, p_{n}\right)$. In particular, if $j=n$, then $f\left(r^{n}\right)=f\left(p_{1}, \ldots, p_{n}\right)$.

(A5) (Uniqueness) If $p \in F\left(p_{1}, \ldots, p_{n}\right)$ and $q \in F\left(q_{1}, \ldots, q_{n}\right)$ satisfy

$$
p_{1} \preceq q_{1} \preceq \ldots \preceq p_{i} \preceq q_{i} \prec p \prec q \prec p_{i+1} \preceq q_{i+1} \preceq \ldots \preceq p_{n} \preceq q_{n}\left(\prec p_{1}\right)
$$

where $0 \leq i \leq n$, then $f\left(p_{1}, \ldots, p_{n}\right)=f\left(q_{1}, \ldots, q_{n}\right)$ holds.

(A6) (Total Multiplicity) The total multiplicity of $f\left(p_{1}, \ldots, p_{n}\right)$ is greater or equal to $2 n+$ 2 for all $\left(p_{1}, \ldots, p_{n}\right) \in I_{\left(\iota_{a}, \iota_{b}\right)}^{n}$ satisfying $f\left(p_{1}, \ldots, p_{n}\right)(a)<2 \iota_{a}$ and $f\left(p_{1}, \ldots, p_{n}\right)(b)<$ $2 \iota_{b}$.

(A7) (Semicontinuity) Let $\left(p_{1, k}, \ldots, p_{n, k}\right)$ be a sequence in $I_{\left(\iota_{a}, \iota_{b}\right)}^{n}$ that converges to the element $\left(p_{1}, \ldots, p_{n}\right) \in I_{\left(\iota_{a}, \iota_{b}\right)}^{n}$ where $p_{1} \in I^{\circ}$. Assume

$$
f\left(p_{1, k}, \ldots, p_{n, k}\right)\left(p_{1, k}\right) \geq 2 \ell
$$

for all $k$. Then

$$
f\left(p_{1}, \ldots, p_{n}\right)\left(p_{1}\right) \geq 2 \ell .
$$

Assume $n \geq 2$. If $p_{1}=p_{2}$ and $p_{1, k} \neq p_{2, k}$ for all $k$, then

$$
f\left(p_{1}, \ldots, p_{n}\right)\left(p_{1}\right) \geq 2 \ell+2
$$

(A8) (Boundary isolation) If $\iota_{a}>1$ (resp. $\iota_{b}>1$ ) and $0<f\left(p_{1}, \ldots, p_{n}\right)(a)<2 \iota_{a}$ $\left(\right.$ resp. $\left.0<f\left(p_{1}, \ldots, p_{n}\right)(b)<2 \iota_{b}\right)$, then $a\left(\right.$ resp. b) is isolated in $F\left(p_{1}, \ldots, p_{n}\right)$.

Proof. Axioms (A1), (A2) and (A3) are trivially true for $f$.

We now prove that $f$ satisfies the Exchangeability Axiom (A4). So we assume for $r \in I$ that

$$
f\left(p_{1}, \ldots, p_{n}\right)(r) \geq 2 j
$$

and $r \neq p_{1}, \ldots, p_{n-j}$. It follows from the definition of $f_{u}$ that $\left(p_{1}, \ldots, p_{n-j}, r^{j}\right) \in I_{\left(\iota_{a}, \iota_{b}\right)}^{n}$. We need to prove that $\varphi_{\left(p_{1}, \ldots, p_{n-j}, r^{j}\right)}=\varphi_{\left(p_{1}, \ldots, p_{n}\right)}$. It is clear that $\varphi\left(p_{1}, \ldots, p_{n}\right)$ lies in $\Lambda_{u}\left(p_{1}, \ldots, p_{n-j}, r^{j}\right)$. Hence

$$
\varphi_{\left(p_{1}, \ldots, p_{n}\right)} \geq \varphi_{\left(p_{1}, \ldots, p_{n-j}, r^{j}\right)} \geq u \text {. }
$$

Since we can squeeze $\varphi_{\left(p_{1}, \ldots, p_{n-j}, r^{j}\right)}$ between $\varphi_{\left(p_{1}, \ldots, p_{n}\right)}$ and $u$ we have that $\varphi_{\left(p_{1}, \ldots, p_{n-j}, r^{j}\right)}$ lies $\Lambda_{u}\left(p_{1}, \ldots, p_{n}\right)$. Hence

$$
\varphi_{\left(p_{1}, \ldots, p_{n-j}, r^{j}\right)} \geq \varphi_{\left(p_{1}, \ldots, p_{n}\right)} \geq u \text {. }
$$


It follows that $\varphi_{\left(p_{1}, \ldots, p_{n-j}, r^{j}\right)}=\varphi_{\left(p_{1}, \ldots, p_{n}\right)}$ and hence

$$
f\left(p_{1}, \ldots, p_{n-j}, r^{j}\right)=f\left(p_{1}, \ldots, p_{n}\right),
$$

and Axiom (A4) follows.

To prove that the Uniqueness Axiom (A5) is satisfied, assume that $p \in F\left(p_{1}, \ldots, p_{n}\right)$ and $q \in F\left(q_{1}, \ldots, q_{n}\right)$ satisfy

$$
p_{1} \preceq q_{1} \preceq \ldots \preceq p_{i} \preceq q_{i} \prec p \prec q \prec p_{i+1} \preceq q_{i+1} \preceq \ldots \preceq p_{n} \preceq q_{n}\left(\prec p_{1}\right)
$$

where $0 \leq i \leq n$. Then the function

$$
\varphi_{\left(p_{1}, \ldots, p_{n}\right)}-\varphi_{\left(q_{1}, \ldots, q_{n}\right)}
$$

has more than $2 n+1$ zeros counted with multiplicities, implying that it vanishes identically. Hence we have $f\left(p_{1}, \ldots, p_{n}\right)=f\left(q_{1}, \ldots, q_{n}\right)$, proving the axiom.

To prove Axiom (A6), first notice that the total multiplicity of $f$ is by definition greater or equal equal to $2 n$. Assume that the total multiplicity of $f$ is equal to $2 n$. Since $f\left(p_{1}, \ldots, p_{n}\right)(a)<2 \iota_{a}$ and $f\left(p_{1}, \ldots, p_{n}\right)(b)<2 \iota_{b}, u$ is $C^{2 \iota_{a}-1}$ at $a$ (resp. $C^{2 \iota_{b}-1}$ at $b$ ) and $a$ (resp. $b$ ) occurs less than $\iota_{a}$ times (resp. $\iota_{b}$ times) as a component of $\left(p_{1}, \ldots, p_{n}\right)$. The function $\varphi_{\left(p_{1}, \ldots, p_{n}\right)}-u \geq 0$ and has precisely $2 n$ zeros counted with multiplicities. More precisely, the set of zeros of $\varphi_{\left(p_{1}, \ldots, p_{n}\right)}-u \geq 0$ is $\left\{p_{1}, \ldots, p_{n}\right\}$, the first $2 \mu_{i}-1$ derivatives vanish in $p_{i}$ for all $i=1, \ldots, n$, and the $2 \mu_{i}$-th derivative is positive in $p_{i}$. Let $\varphi_{2}$ be as in the proof of Lemma 2.3. The $2 \mu_{i}$-th derivative of $\varphi_{2}$ is positive in $p_{i}$ for all $i=1, \ldots, n$. It follows that there is a sufficiently large $m$ such that

$$
\varphi_{\left(p_{1}, \ldots, p_{n}\right)}-u \geq \frac{1}{m} \varphi_{2} \geq 0 .
$$

Hence

$$
\varphi_{\left(p_{1}, \ldots, p_{n}\right)}-\frac{1}{m} \varphi_{2} \in \Lambda_{u}\left(p_{1}, \ldots, p_{n}\right),
$$

contradicting the definition of $\varphi_{\left(p_{1}, \ldots, p_{n}\right)}$.

We now prove the Semicontinuity Axiom (A7). Let $\left(p_{1, k}, \ldots, p_{n, k}\right)$ be a sequence in $I_{\left(\iota_{a}, \iota_{b}\right)}^{n}$ that converges to the element $\left(p_{1}, \ldots, p_{n}\right) \in I_{\left(\iota_{a}, \iota_{b}\right)}^{n}$ where $p_{1} \in I^{\circ}$. Assume that

$$
f\left(p_{1, k}, \ldots, p_{n, k}\right)\left(p_{1, k}\right) \geq 2 \ell
$$

for all $k$. Then clearly

$$
f\left(p_{1}, \ldots, p_{n}\right)\left(p_{1}\right) \geq 2 \ell,
$$

since $\varphi_{\left(p_{1, k}, \ldots, p_{n, k}\right)}$ converges to $\varphi_{\left(p_{1}, \ldots, p_{n}\right)}$ together with all its derivatives. Now assume that $n \geq 2, p_{1}=p_{2}$ and $p_{1, k} \neq p_{2, k}$ for all $k$. We need to prove that

$$
f\left(p_{1}, \ldots, p_{n}\right)\left(p_{1}\right) \geq 2 \ell+2 .
$$

We consider the sequence $\left(v_{k}\right)$ where $v_{k}=\varphi_{\left(p_{1, k}, \ldots, p_{n, k}\right)}-u$. Notice that the first derivative of $v_{k}$ vanishes in $p_{1, k}$ and $p_{2, k}$ for all $k$. Hence there is for every $k$ a point $q_{2, k}$ between $p_{1, k}$ 
and $p_{2, k}$ such that the second derivative of $v_{k}$ vanishes in $q_{2, k}$. If $\ell \geq 2$, then the second derivative of $v_{k}$ vanishes in $p_{1, k}$ and $q_{2, k}$ and there must be a point $q_{3, k}$ between $p_{1, k}$ and $q_{2, k}$ in which the third derivative of $v_{k}$ vanishes. We continue this argument inductively and show that there is for every $k$ a point $q_{2 \ell, k}$ between $p_{1, k}$ and $q_{2 \ell, k}$ such that the $2 \ell$-th derivative of $v_{k}$ vanishes. The sequence $\left(v_{k}\right)$ converges with all its derivatives to $v=\varphi_{\left(p_{1}, \ldots, p_{n}\right)}-u$ and the sequence $\left(q_{2 \ell, k}\right)$ converges to $p$. It follows that at least the $2 \ell$ first derivatives of $\varphi\left(p_{1}, \ldots, p_{n}\right)$ and $u$ coincide in $p_{1}$. Since $u \leq \varphi_{\left(p_{1}, \ldots, p_{n}\right)}$ also the $2 \ell+1$-st derivatives coincide in $p$. Hence $f\left(p_{1}, \ldots, p_{n}\right)\left(p_{1}\right) \geq 2 \ell+2$ which finishes the proof of Axiom (A7).

Finally we prove (A8). Suppose $\iota_{a}>1$ and $0<f\left(p_{1}, \ldots, p_{n}\right)(a)<2 \iota_{a}$. Suppose also that $a$ is not isolated in $F\left(a_{1}, \ldots, a_{n}\right)$. There is a sequence $\left(q_{n}\right)$ in $F\left(a_{1}, \ldots, a_{n}\right)$ such that $\lim _{n \rightarrow \infty} q_{n}=a$. Then we have

$$
\varphi_{\left(p_{1}, \ldots, p_{n}\right)}\left(q_{n}\right)=u\left(q_{n}\right)
$$

for all $n$. Since $u(t)$ is $C^{2 \iota_{a}-1}$ at $a$ this implies that

$$
\varphi_{\left(p_{1}, \ldots, p_{n}\right)}(a)=u(a)
$$

and

$$
\varphi_{\left(p_{1}, \ldots, p_{n}\right)}^{(j)}(a)=u^{(j)}(a)
$$

for $j=1,2, \ldots, 2 \iota_{a}-1$. It follows that $f\left(p_{1}, \ldots, p_{n}\right)(a)=2 \iota_{a}$, a contradiction. Hence $a$ is isolated in $F\left(a_{1}, \ldots, a_{n}\right)$.

We now give the following definition.

2.5 Definition. Let $I$ either be the whole circle $S^{1}$ or a proper closed interval on $S^{1}$. In the second case we assume we have a pair of $\left(\iota_{a}, \iota_{b}\right)$ of nonnegative integers which are less than or equal to $\infty$. A map

$$
f: I_{\left(\iota_{a}, \iota_{b}\right)}^{n} \rightarrow \operatorname{Map}\left(S^{1}, 2 \mathbf{N}_{0} \cup\{\infty\}\right) .
$$

is called an intrinsic system of order $2 n+1$ on I (satisfying the boundary regularity condition $\left.\left(\iota_{a}, \iota_{b}\right)\right)$ if it satisfies the axioms (A1) to (A8) in Proposition 2.4. (If $I$ is the whole circle one should of course delete everything referring to the boundary conditions in the axioms.) A point $s \in S^{1}$ is called an $f$-flex if $f\left(s^{n}\right)(s) \geq 2 n+2$. Moreover, if $F\left(s^{n}\right)$ is connected, it is called a clean $f$-flex.

The map $f_{u}$ as in Proposition 2.4 is of course an example of an intrinsic system of order $2 n+1$, and an $f_{u}$-flex is nothing but a global $\mathcal{A}$-flex.

Notice that the values of $f\left(p_{1}, \ldots, p_{n}\right)$ can be finite numbers greater than $2 n$ although this does not happen for $f_{u}$ by definition. This will for example happen in the course of the reduction procedure introduced in Lemma 3.7 that we will frequently apply in the paper.

We next give two more examples of intrinsic systems that come from curve theory.

2.6 Example. (i) Let $\gamma: S^{1} \rightarrow \mathbf{R}^{2}$ be a simple closed regular $C^{2}$-curve. For an arbitrary circle $C$, we associate a function $\mu_{C}(r)$ on $S^{1}$ that maps a point $r$ on $\gamma$ to the multiplicity 
with which $C$ and $\gamma$ meet in $r$. The function $\mu_{C}(r)$ takes values in $\{0,1,2,3\}$ since we are only assuming the curve to be $C^{2}$-regular. The value of $\mu_{C}(r)$ is of course zero in points in which $C$ and $\gamma$ do not meet. We let $C_{p}^{\bullet}\left(p \in S^{1}\right)$ (resp. $\left.C_{p}^{\circ}\right)$ denote the uniquely defined maximal inscribed (resp. minimal circumscribed) circle that is tangent to $\gamma$ in $p$. We set

$$
f_{1}^{\bullet}(p, q)(r)= \begin{cases}\mu_{C_{p, q}}(r) & \text { if } \mu_{C_{p, q}^{\bullet}}(r) \leq 2 \\ \infty & \text { if } \mu_{C_{p, q}^{\bullet}}(r) \geq 3\end{cases}
$$

We define the map $f_{1}^{\circ}$ similarly. One can easily verify that $f_{1}^{\bullet}$ and $f_{1}^{\circ}$ are both intrinsic systems of order 3. (Notice that the dimension of the space of circles in the Euclidean plane is three.) A point $p$ is called a clean maximal (resp. minimal) vertex if the osculating circle $C_{p}$ is inscribed (resp. circumscribed) and meets the curve in a connected set. When $\gamma$ is $C^{3}$, the critical points of the curvature function of the curve are called vertices. For a $C^{3}$ regular curve, clean vertices are vertices of the curve. However the vertices of a curve have a priori no such global properties. The concept of an intrinsic system is designed to find vertices with such global properties. It is well known and can be proved with the methods of this paper that there are at least four clean vertices on a curve $\gamma$ as above. The notion of an 'intrinsic circle system' as a family of closed subset $\left(F_{p}\right)_{p \in S^{1}}$ in $S^{1}$ satisfying certain axioms, see Section six below, was introduced in [Um]. Several applications were given in [Um] and [TU1]. The family of supports $\left(F_{1}^{\bullet}(p)\right)_{p \in S^{1}}$ and $\left(F_{1}^{\circ}(p)\right)_{p \in S^{1}}$ of the intrinsic systems $f_{1}^{\bullet}$ and $f_{1}^{\circ}$ introduced above satisfy the axioms of an intrinsic circle system. As a consequence, when the curve has finitely many maximal and minimal vertices, one can prove that it satisfies a Bose type formula as mentioned in the introduction; see [Um].

(ii) Let $\gamma$ be a strictly convex $C^{4}$-curve in the real projective plane $P^{2}$. We identify $\gamma$ with $S^{1}$. Let $\Gamma$ be a nondegenerate conic in $P^{2}$. Then we associate to $\Gamma$ a function $\mu_{\Gamma}(r)$ on $S^{1}$ that maps a point $r$ on $\gamma$ to the multiplicity with which $\Gamma$ and $\gamma$ meet in $r$. The function $\mu_{\Gamma}(r)$ takes values in $\{0,1, \ldots, 5\}$ since we are only assuming the curve to be $C^{4}$ regular. The value of $\mu_{\Gamma}(r)$ is of course zero in points in which $\Gamma$ and $\gamma$ do not meet. Let $(p, q) \in S^{2}$. If $p \neq q$, we let $\Gamma_{p, q}^{\bullet}\left(\operatorname{resp} . \Gamma_{p, q}^{\circ}\right)$ denote the uniquely defined maximal inscribed (resp. minimal circumscribed) conic that is tangent to $\gamma$ in $p$ and $q$. If $p=q$, we let $\Gamma_{p, q}^{\bullet}$ (resp. $\Gamma_{p, q}^{\circ}$ ) denote the uniquely defined maximal inscribed (resp. minimal circumscribed) conic that meets $\gamma$ with multiplicity at least four in $p=q$. We set

$$
f_{2}^{\bullet}(p, q)(r)= \begin{cases}\mu_{\Gamma_{p, q}^{\bullet}}(r) & \text { if } \mu_{\Gamma_{p, q}^{\bullet}}(r) \leq 4 \\ \infty & \text { if } \mu_{\Gamma_{p, q}^{\bullet}}(r) \geq 5\end{cases}
$$

We define the map $f_{2}^{\circ}$ similarly. One can easily verify that $f_{2}^{\bullet}$ and $f_{2}^{\circ}$ are both intrinsic systems of order 5. (Notice that the dimension of the space of conics in $P^{2}$ is five.) If an osculating conic at a point $p$ is inscribed (resp. circumscribed) and meets the curve $\gamma$ in a connected set, we call $p$ a clean maximal sextactic point (resp. clean minimal sextactic point). When $\gamma$ is $C^{5}$, a point where the osculating conic meets with multiplicity greater than 5 is called a sextactic point. By (A6), the clean maximal (resp. minimal) sextactic points are sextactic points of $\gamma$ whenever the curve is $C^{5}$. Existence of six sextactic points where the osculating conics are inscribed or circumscribed was proved by Mukhopadhyaya [Mu2]; see also [TU2] for an alternative proof. These sextactic points might however not be clean. We will refine the methods of [TU2] and prove in Theorem 5.3 below the existence of at least six clean sextactic points on the curve $\gamma$. 
In [TU2] we introduced the concept of an intrinsic conic system to prove the above mentioned theorem of Mukhopadhyaya and more generally to find sextactic points on simple closed curves in $P^{2}$. Intrinsic conic systems are very similar to intrinsic systems of order five.

We now generalize the construction of an intrinsic system of order $2 n+1$ on $I$ associated to a function $u$ by taking base points $c_{1}, \ldots, c_{r}$ into account since that will be needed in Section five. Let $\nu_{1}, \ldots, \nu_{r}$ be positive integers. We set $N=n+m$ where $m=\sum_{h=1}^{r} \nu_{h}$ and let $\mathcal{A}$ denote a Chebyshev space of order $2 N+1$. Let $I$ be a closed interval not containing the base points $c_{1}, \ldots, c_{r}$. We assume we have a function $u$ that is piecewise $C^{2 n}$. We assume furthermore that $u$ is $C^{2 n}$ on $I$ satisfying the boundary regularity condition $\left(\iota_{a}, \iota_{b}\right)$; see Definition 2.2. We now generalize Lemma 2.3 to this new situation.

2.7 Lemma. Assume the function $u$ and the base points $c_{1}, \ldots, c_{r}$ to be as described before this lemma. We let $\mu_{i}\left(\right.$ resp. $\left.\nu_{h}\right)$ denote the multiplicity with which $p_{i}$ (resp. $c_{h}$ ) occurs as a component of the $n$-uple $\left(p_{1}, \ldots, p_{n}\right)$ (resp. m-uple $\left.\left(c_{1}, \ldots, c_{m}\right)\right)$. Suppose also that $u(t)$ be at least $C^{2 \nu_{h}}$ on some neighborhood of $c_{h}$ for all $h=1, \ldots, m$. For $\left(p_{1}, \ldots, p_{n}\right) \in I_{\left(\iota_{a}, \iota_{b}\right)}^{n}$ we let $\hat{\Lambda}$ denote the one-dimensional set of functions $\varphi \in \mathcal{A}$ such that

$$
\begin{array}{ll}
\varphi^{(k)}\left(p_{j}\right)=u^{(k)}\left(p_{j}\right) & \text { for } k=0, \ldots, 2 \mu_{j}-1 \text { and } j=1, \ldots, n \\
\varphi^{(\ell)}\left(c_{h}\right)=u^{(\ell)}\left(c_{h}\right) & \text { for } \ell=0, \ldots, 2 \nu_{h}-1 \text { and } h=1, \ldots, m .
\end{array}
$$

Then the subset of functions $\varphi \in \hat{\Lambda}$ such that $\varphi \geq u$ is a nonempty closed interval that we denote by $\hat{\Lambda}_{u}\left(p_{1}, \ldots, p_{n}\right)$.

Proof. One can proceed exactly as in the proof of Lemma 2.3.

For a point $\left(p_{1}, \ldots, p_{n}\right) \in I_{\left(\iota_{a}, \iota_{b}\right)}^{n}$ we define the function $\hat{\varphi}_{\left(p_{1}, \ldots, p_{n}\right)} \in \hat{\Lambda}_{u}\left(p_{1}, \ldots, p_{n}\right)$ by setting

$$
\hat{\varphi}_{\left(p_{1}, \ldots, p_{n}\right)}(t)=\inf \left\{\varphi(t) \in \hat{\Lambda}_{u}\left(p_{1}, \ldots, p_{n}\right)\right\} .
$$

As above we define a map

$$
\hat{f}_{u}: I^{n} \rightarrow \operatorname{Map}\left(S^{1}, 2 \mathbf{N}_{0} \cup\{\infty\}\right),
$$

satisfying the same first five conditions (i) to (v) and the following three new conditions $\left(\mathrm{vi}^{\prime}\right)$, (vii) and (viii) (with (vi') replacing the previous condition (vi)):

$$
\hat{f}_{u}\left(p_{1}, \ldots, p_{n}\right)(q)=2
$$

if $q \notin I \cup\left\{c_{1}, \ldots, c_{r}\right\}$ and $u(q)=\hat{\varphi}_{\left(p_{1}, \ldots, p_{n}\right)}(q)$;

$$
\hat{f}_{u}\left(p_{1}, \ldots, p_{n}\right)(q)=0
$$

if $q=c_{h}$ for some $h=1, \ldots, r$ and precisely $2 \nu_{h}-1$ derivatives of $u$ and $\hat{\varphi}_{\left(p_{1}, \ldots, p_{n}\right)}$ agree in $q$; and finally

$$
\hat{f}_{u}\left(p_{1}, \ldots, p_{n}\right)(q)=2
$$


if $q=c_{h}$ for some $h=1, \ldots, r$ and more than $2 \nu_{h}-1$ derivatives of $u$ and $\hat{\varphi}_{\left(p_{1}, \ldots, p_{n}\right)}$ agree in $q$.

2.8 Proposition. The map $\hat{f}_{u}$ is an intrinsic system of order $2 n+1$. We shall call it the intrinsic system of order $2 n+1$ with base points $c_{1}, \ldots c_{r}$ associated to $u$.

Proof. The proposition can be proved by modifying the proof of Proposition 2.4.

\section{$\S 3$ First consequences of the axioms of an intrinsic system}

In this section, we shall derive some first consequences of the axioms of intrinsic systems. It should be remarked that Lemmas 3.3 to 3.6 below are rather easy to check if the intrinsic system $f=f_{u}$ comes from a periodic function $u$. Still we shall prove them only using the axioms since they are also important for our applications to sextactic points in Section five.

The following trivial lemma will frequently be used, mostly without saying so explicitly.

3.1. Lemma. Let $J$ be a closed subinterval of the interval $I$. Let $f$ be an intrinsic system of order $2 n+1$ on I satisfying the boundary regularity condition $\left(\iota_{a}, \iota_{b}\right)$ if $I$ is not the whole circle. Then the restriction of $f$ to $J^{n} \cap I_{\left(\iota_{a}, \iota_{b}\right)}^{n}$ is an intrinsic system of order $2 n+1$ on $J$ satisfying the boundary regularity condition $\left(n, \iota_{b}\right)$ if a is not in $J$ and $b$ is in $J$, the condition $\left(\iota_{a}, n\right)$ if $b$ is not in $J$ and $a$ is in $J$, and $(n, n)$ if neither a nor $b$ lies in $J$.

The following lemma is an immediate consequence of Axiom (A6).

3.2. Lemma. If $p \in I^{\circ}$ and $F(p, \ldots, p)$ consists only of the point $p$, then $p$ is an f-flex.

The Exchangeability Axiom (A4) immediately implies the following lemma.

3.3. Lemma. If $f\left(p_{1}, \ldots, p_{n}\right)(p) \geq 2 n+2$ for a point $p \in I$, then $p$ is an $f$-flex.

The next lemma is an application of the semicontinuity Axiom (A7).

3.4. Lemma. (The Multiplicity Lemma) We have $f\left(p^{j}, p_{j+1}, \ldots, p_{n}\right)(p) \geq 2 j$ for every $p \in I^{\circ}$.

Proof. Let $\left(p_{l, k}\right)$ for $l=1, \ldots, j$ be $j$ sequences in $I$ that converge to $p$ and assume that $p_{l, k} \neq p$ for all $l$ and all $k$. Axioms (A3) and (A7) imply that

$$
f\left(p^{2}, p_{3, k}, \ldots, p_{j, k}, p_{j+1}, \ldots, p_{n}\right)(p) \geq 4
$$

for every $k$. (Here we fixed $k$ in the third and later arguments and let $k$ in the first two arguments go to infinity when applying (A7).) We can now use the Symmetry Axiom (A2) to bring $p_{3, k}$ into the second slot and use (A7) again to prove

$$
f\left(p^{3}, p_{4, k}, \ldots, p_{j, k}, p_{j+1}, \ldots, p_{n}\right)(p) \geq 6
$$

for all $k$. We continue this argument inductively until we have proved the lemma. 
3.5. Lemma. If $r \in F\left(p_{1}, \ldots, p_{n}\right) \cap I^{\circ}$ is not isolated in $F\left(p_{1}, \ldots, p_{n}\right) \cap I^{\circ}$, then $r$ is an $f$-flex.

Proof. We assume that $f\left(p_{1}, \ldots, p_{n}\right)(r)$ is a finite number $k$. Set $p_{1, k}=r$. Let $\left(p_{2, k}\right)$ be a sequence in $F\left(p_{1}, \ldots, p_{n}\right)$ of pairwise different points that are all different from $r$ and converge to $r$. After possibly permuting and relabeling the points $p_{1}, \ldots, p_{n}$, we have by the Exchangeability Axiom (A4) that $f\left(p_{1}, \ldots, p_{n}\right)(r)=f\left(p_{1, k}, p_{2, k}, p_{3}, \ldots, p_{n}\right)\left(p_{1, k}\right)=k$. Now the Semicontinuity Axiom (A7) implies that $f\left(p_{1}, \ldots, p_{n}\right)(r)>k$, a contradiction. Hence $f\left(p_{1}, \ldots, p_{n}\right)(r)=\infty$. It now follows from the Exchangeability Axiom (A4) that $f\left(r^{n}\right)(r)=\infty$.

The next lemma is the starting point of the idea of an intrinsic system and the main tool in the paper [Um]. Notice that the Semicontinuity Axiom (A7) is not used in its proof. The idea behind the lemma goes back to $\mathrm{H}$. Kneser [Kn]. Therefore we would like to call it the Kneser Lemma although it is strictly speaking not due to him.

3.6. Lemma. (The Kneser Lemma) Let $f$ be an intrinsic system of order three on $I=[a, b]$ satisfying the boundary regularity condition $\left(\iota_{a}, \iota_{b}\right)$ with $\iota_{a}, \iota_{b} \geq 1$. Suppose that $a, b \in F(a)$ and $F(a) \cap(a, b)$ is empty. Then there exists a point $c \in(a, b)$ such that $F(c)$ is connected and contained in $(a, b)$. In particular, $c$ is an $f$-flex.

Proof. Let $q$ be any point in the interval $(a, b)$. Then the Uniqueness Axiom (A5) implies that $F(q)$ is contained in $[a, b]$ and the Exchangeability Axiom (A4) implies that $F(q)$ cannot contain $a$ and $b$. Hence $F(q) \subset(a, b)$. Let $c_{1}$ be the midpoint of the interval $[a, b]$. If $F\left(c_{1}\right)$ is connected then the proof is finished. If $F\left(c_{1}\right)$ is not connected, there are two different points $a_{1}, b_{1} \in F\left(c_{1}\right)$ such that $F\left(c_{1}\right) \cap\left(a_{1}, b_{1}\right)$ is empty. Notice that the length of $\left[a_{1}, b_{1}\right]$ is less than half the length of $[a, b]$ and $F(q) \subset\left(a_{1}, b_{1}\right)$ for every $q \in\left(a_{1}, b_{1}\right)$. Let $c_{2}$ be the midpoint of $\left[a_{1}, b_{1}\right]$. Then $F\left(c_{2}\right) \subset\left(a_{1}, b_{1}\right)$. If $F\left(c_{2}\right)$ is connected we have finished the proof. If not, we continue inductively and find a nested sequence of intervals $\left[a_{n}, b_{n}\right]$ with midpoints $c_{n+1}$ such that $a_{n}, b_{n} \in F\left(c_{n}\right)$ and $F\left(c_{n}\right) \cap\left(a_{n}, b_{n}\right)$ is empty. Furthermore, the length of $\left[a_{n}, b_{n}\right]$ is less than $(1 / 2)^{n}$ the length of $[a, b]$. We have $F(q) \subset\left[a_{n}, b_{n}\right]$ for all $q \in\left(a_{n}, b_{n}\right)$. We stop the induction if we arrive at a connected set $F\left(c_{n+1}\right)$. Otherwise we observe that the sequence $\left(c_{n}\right)$ converges to a point $c$. Then $F(c)$ consists of $c$ only since $F(c) \subset\left(a_{n}, b_{n}\right)$ for all $n$. It now follows from Lemma 3.2 that $c$ is an $f$-flex.

The main strategy in finding an $f$-flex of an intrinsic system of order $2 n+1$ is to reduce the order inductively until we can apply the Kneser Lemma. We now start explaining this procedure.

Assume $n \geq 2$ and let $f$ be an intrinsic system of order $2 n+1$ on $I=[a, b]$ satisfying the boundary regularity condition $\left(\iota_{a}, \iota_{b}\right)$. We choose $r=a$ (or $\left.r=b\right)$ and assume that $\iota_{a} \geq 2\left(\right.$ or $\left.\iota_{b} \geq 2\right)$. If $r=a$, let $\left(p_{1}, \ldots, p_{n-1}\right) \in I_{\left(\iota_{a}-1, \iota_{b}\right)}^{n-1}$ and let $q \in S^{1}$. We set

$$
f_{r}\left(p_{1}, \ldots, p_{n-1}\right)(q)= \begin{cases}f\left(r, p_{1}, \ldots, p_{n-1}\right)(q) & \text { if } q \neq r \\ f\left(r, p_{1}, \ldots, p_{n-1}\right)(r)-2 & \text { if } q=r\end{cases}
$$

where we of course use the convention that $\infty-2=\infty$. We define $f_{r}$ analogously on $I_{\left(\iota_{a}, \iota_{b}-1\right)}^{n-1}$ if $r=b$. 
3.7. Lemma. Let $I=[a, b]$ be a closed interval on $S^{1}$ and $f$ an intrinsic system of order $2 n+1$ on $I$ for some $n \geq 2$ satisfying the boundary regularity condition $\left(\iota_{a}, \iota_{b}\right)$. Let $r$ be an endpoint of $I$ and assume that $\iota_{r} \geq 2$. Then $f_{r}$ is an intrinsic system of order $2 n-1$ on $I$ satisfying the boundary regularity condition $\left(\iota_{a}-1, \iota_{b}\right)$ if $r=a$ and $\left(\iota_{a}, \iota_{b}-1\right)$ if $r=b$.

Remark. The restriction in the Semicontinuity Axiom (A7) that $p_{1}$ be in the interior $I^{\circ}$ of $I$ comes from the fact that otherwise we would not be able to prove that $f_{r}$ satisfies that axiom.

Proof. We assume throughout the proof that $r=a$. The case $r=b$ is completely analogous.

First notice that $f\left(r, p_{1}, \ldots, p_{n-1}\right)(r) \geq 2$ for all $\left(p_{1}, \ldots, p_{n-1}\right) \in I_{\left(\iota_{a}-1, \iota_{b}\right)}^{n-1}$ by Axiom (A3). It follows that the values of $f_{r}\left(p_{1}, \ldots, p_{n-1}\right)$ are nonnegative.

To see that (A1) is satisfied for $f_{r}$ we remark that the sets $F\left(r, p_{1}, \ldots, p_{n-1}\right)$ and $F_{r}\left(p_{1}, \ldots, p_{n-1}\right)$ are equal and hence both closed if $f\left(r, p_{1}, \ldots, p_{n-1}\right)(r)>2$. We have that $F_{r}\left(p_{1}, \ldots, p_{n-1}\right)=F\left(r, p_{1}, \ldots, p_{n-1}\right)-\{r\}$ if $f\left(r, p_{1}, \ldots, p_{n-1}\right)(r)=2$. Then (A8) implies that $r$ is isolated in $F\left(r, p_{1}, \ldots, p_{n-1}\right)$ since $\iota_{r} \geq 2$. Hence $F_{r}\left(p_{1}, \ldots, p_{n-1}\right)$ is also closed in this case.

Axioms (A2) and (A3) for $f$ clearly imply Axioms (A2) and (A3) for $f_{r}$.

To prove (A4) for $f_{r}$, assume that $f_{r}\left(p_{1}, \ldots, p_{n-1}\right)(q) \geq 2 j$ for $q \in I$ and $q$ does not coincide with any of the $p_{1}, \ldots, p_{n-j-1}$ where $j \leq n-1$. First assume that $q \neq r$. The we have $f\left(r, p_{1}, \ldots, p_{n-1}\right)(q) \geq 2 j$ and Axiom (A4) for $f$ implies that

$$
f\left(r, p_{1}, \ldots, p_{n-j-1}, q^{j}\right)=f\left(r, p_{1}, \ldots, p_{n-1}\right)
$$

Hence

$$
f_{r}\left(p_{1}, \ldots, p_{n-j-1}, q^{j}\right)=f_{r}\left(p_{1}, \ldots, p_{n-1}\right)
$$

If $q=r$ we have $f\left(p_{1}, \ldots, p_{n-1}, r\right)(q) \geq 2 j+2$. By Axiom (A4) for $f$ this implies that

$$
f\left(p_{1}, \ldots, p_{n-j-1}, q^{j}, r\right)=f\left(p_{1}, \ldots, p_{n-1}, r\right)
$$

Hence we again have that $f_{r}\left(p_{1}, \ldots, p_{n-j-1}, q^{j}\right)=f_{r}\left(p_{1}, \ldots, p_{n-1}\right)$.

Axiom (A5) for $f_{r}$ follows immediately from Axiom (A5).

Axiom (A6) for $f_{r}$ follows easily from Axiom (A6) for $f$ since the total multiplicity of $f_{r}\left(p_{1}, \ldots, p_{n-1}\right)$ is two less than the one of $f\left(r, p_{1}, \ldots, p_{n-1}\right)$ if the latter number is finite. If the total multiplicity of $f\left(r, p_{1}, \ldots, p_{n-1}\right)$ is infinite, then the same is true for $f_{r}\left(p_{1}, \ldots, p_{n-1}\right)$.

We now prove $(\mathrm{A} 7)$ for $f_{r}$. Let $\left(p_{1, k}, \ldots, p_{n-1, k}\right)$ be a sequence in $I_{\left(\iota_{a}-1, \iota_{b}\right)}^{n-1}$ that converges to the element $\left(p_{1}, \ldots, p_{n-1}\right)$ where $p_{1} \in I^{\circ}$. Notice that $p_{1} \neq r$. Assume

$$
f_{r}\left(p_{1, k}, \ldots, p_{n-1, k}\right)\left(p_{1, k}\right) \geq 2 \ell
$$

Then

$$
f\left(p_{1, k}, \ldots, p_{n-1, k}, r\right)\left(p_{1, k}\right) \geq 2 \ell
$$


for $k$ large. Now (A6) for $f$ immediately implies that

$$
f_{r}\left(p_{1}, \ldots, p_{n-1}\right)\left(p_{1}\right) \geq 2 \ell .
$$

Assume $n \geq 3$. If $p_{1}=p_{2} \neq r$ and $p_{1, k} \neq p_{2, k}$ for all $k$, then Axiom (A7) implies

$$
f_{r}\left(p_{1}, \ldots, p_{n-1}\right)\left(p_{1}\right)=f\left(p_{1}, \ldots, p_{n-1}, r\right)\left(p_{1}\right) \geq 2 \ell+2 .
$$

This shows that $f_{r}$ satisfies Axiom (A7).

Axiom (A8) for $f_{r}$ follows easily from Axiom (A8) for $f$.

3.8. Lemma. Let $I=[a, b]$ be an interval on $S^{1}, f$ an intrinsic system of order $2 n+1$ on I satisfying the boundary regularity condition $\left(n, \iota_{b}\right)$ with $\iota_{b} \geq 1$. Assume that $f\left(a^{n}\right)=$ $f\left(b, a^{n-1}\right)$ and $F\left(a^{n}\right) \cap(a, b)$ is empty. Then there exists an $f$-flex in the open interval $(a, b)$.

Similarly, if $f$ satisfies the boundary regularity condition $\left(\iota_{a}, n\right)$ with $\iota_{a} \geq 1, f\left(b^{n}\right)=$ $f\left(a, b^{n-1}\right)$ and $F\left(b^{n}\right) \cap(a, b)$ is empty, then there exists an $f$-flex in the open interval $(a, b)$.

Proof. We proof the lemma by induction on $n$. The lemma is true for $n=1$ by the Kneser Lemma 3.6. Assume the lemma is true for $n-1 \geq 1$.

Assume that $f\left(a^{n}\right)=f\left(b, a^{n-1}\right)$ and $F\left(a^{n}\right) \cap(a, b)$ is empty where $f$ is an intrinsic system of order $2 n+1$. Then $f_{a}$ is an intrinsic system of order $2 n-1$ by Lemma 3.7. Notice that $f_{a}\left(a^{n-1}\right)=f_{a}\left(b, a^{n-1}\right)$ and $F_{a}\left(a^{n-1}\right) \cap(a, b)=\emptyset$. By the induction hypothesis there is a point $c \in(a, b)$ that is an $f_{a}$-flex with respect to $f_{a}$. This implies that $f\left(a, c^{n-1}\right)(c) \geq 2 n$. By Axiom (A4) for $f$ this implies $f\left(c^{n}\right)=f\left(a, c^{n-1}\right)$. We can assume that $c$ is isolated in $F\left(c^{n}\right)$ since $c$ is otherwise an $f$-flex by Lemma 3.5 and there would be nothing left to prove. Let $d$ be the point in $[a, c) \cap F\left(c^{n}\right)$ closest to $c$. We have $f\left(c^{n}\right)=f\left(d, c^{n-1}\right)$ and $F\left(c^{n}\right) \cap(d, c)=\emptyset$. Then we can again use the induction hypothesis and we find an $f_{c}$-flex $e$ of $f_{c}$ in the interval $(d, c)$. Set $J=[e, c]$.

Let $\mathcal{C}$ denote the set of $(\alpha, \beta) \in J \times J$ such that $\alpha<\beta, f\left(\alpha^{n}\right)=f\left(\beta, \alpha^{n-1}\right)$ and $F\left(\alpha^{n}\right) \cap(\alpha, \beta)=\emptyset$. By arguments as in the previous paragraph we see that $\mathcal{C}$ is nonempty.

We let $\delta_{\alpha, \beta}$ denote the distance between $\alpha$ and $\beta$. Let $\delta$ denote the infimum over $\delta_{\alpha, \beta}$ for $(\alpha, \beta) \in \mathcal{C}$.

We consider a sequence $\left\{\left(\alpha_{k}, \beta_{k}\right)\right\}$ in $\mathcal{C}$ such that $\delta_{\alpha_{k}, \beta_{k}}$ converges to $\delta$. By going to subsequences if necessary, we may assume that

$$
\lim _{k \rightarrow \infty} \alpha_{k}=\alpha, \quad \lim _{k \rightarrow \infty} \beta_{k}=\beta .
$$

If $\alpha=\beta$, then it follows immediately from Axiom (A7) since $\alpha \in J \subset I^{\circ}$ that

$$
f\left(\alpha^{n}\right)(\alpha) \geq 2(n+1)
$$

and we have that $\alpha \in J \subset(a, b)$ is an $f$-flex.

We can therefore assume that $\delta>0$. By (A7) we have $f\left(\beta, \alpha^{n-1}\right)(\alpha) \geq 2 n$ and hence $f\left(\alpha^{n}\right)=f\left(\beta, \alpha^{n-1}\right)$ by the Exchangeability Axiom (A4). We can assume that $\alpha$ and $\beta$ are isolated in $F\left(\alpha^{n}\right)$ since otherwise we have an $f$-flex by Lemma 3.5. Let $\beta^{\prime}$ be the point 
in $F\left(\alpha^{n}\right) \cap(\alpha, \beta]$ closest to $\alpha$. We now argue as in the second paragraph of the proof and find points $\gamma, \delta \in\left(\alpha, \beta^{\prime}\right)$ such that $(\gamma, \delta) \in \mathcal{C}$. Clearly $\delta_{\gamma, \alpha}<\delta$, which is a contradiction. This finishes the proof of the claim in the first paragraph of the lemma. The proof of the claim in the second paragraph is similar.

The following two propositions are the main technical result of this section. Notice that very similar ideas go at least back to Mukhopadhyaya ([Mu1], Propositions I and II) and Haupt and Künneth [HK], p. 47. The main difference between our approach and theirs is that ours is more global in nature and therefore allows us to prove the existence of flexes satisfying global properties like being clean. The name of the propositions is taken from the book $[\mathrm{HK}]$.

3.9. Proposition. (The Contraction Lemma I) Let $I=[a, b]$ be an interval on $S^{1}, f$ an intrinsic system of order $2 n+1$ on I satisfying the boundary regularity condition $\left(\iota_{a}, \iota_{b}\right)$ with $\iota_{a}+\iota_{b}>n$. Let $p_{1}, \ldots, p_{n+1} \in I$ be such that $\left(p_{1}, \ldots, p_{n}\right) \in I_{\left(\iota_{a}, \iota_{b}\right)}^{n}$ and

$$
f\left(p_{1}, \ldots, p_{n}\right)(a)+f\left(p_{1}, \ldots, p_{n}\right)(b) \geq 2(n+1) .
$$

Then there exists an $f$-flex in the open interval $(a, b)$.

Proof. We shall prove the proposition by induction. If $n=1$, it follows from the Kneser Lemma 3.6. We now assume that $n \geq 2$. Then $\iota_{a}+\iota_{b}>n$ implies that $\iota_{a} \geq 2$ or $\iota_{b} \geq 2$. We consider the case $\iota_{a} \geq 2$ (the case $\iota_{b} \geq 2$ being similar). By Lemma 3.7, $f_{a}$ is an intrinsic system of order $2 n-1$ on $I$ satisfying the boundary regularity condition $\left(\iota_{a}-1, \iota_{b}\right)$. By the induction hypothesis, we find an $f_{a}$-flex $s$ on $(a, b)$. Then by the definition of $f_{a}\left(s^{n-1}\right)$, we have $f\left(a, s^{n-1}\right)(s) \geq 2 n$ and hence $f\left(s^{n}\right)=f\left(a, s^{n-1}\right)$. If $s$ is not isolated in $F\left(a, s^{n-1}\right)$, then $s$ is an $f$-flex by Lemma 3.5. We can therefore assume that $s$ is isolated in $F\left(a, s^{n-1}\right)$ and let $c$ be the point closest to $s$ in $[a, s) \cap F\left(a, s^{n-1}\right)$. We get

$$
f\left(s^{n}\right)=f\left(c, s^{n-1}\right) \quad \text { and } \quad F\left(s^{n}\right) \cap(c, s)=\emptyset .
$$

Now by Lemma 3.8, we find an $f$-flex on $(c, s) \subset(a, b)$.

3.10. Proposition. (The Contraction Lemma II) Let $f$ be an intrinsic system of order $2 n+1$ on $I=[a, b]$ satisfying. Let $p_{1}, \ldots, p_{n+1} \in I^{\circ}$ be such that $p_{1} \preceq \ldots \preceq p_{n+1}$ and

$$
\sum_{t \in\left\{p_{1}, \ldots, p_{n+1}\right\}} f\left(p_{1}, \ldots, p_{n}\right)(t) \geq 2(n+1)
$$

(Notice that repeated points in the sequence $p_{1}, \ldots, p_{n+1}$ only enter once into the sum.) Then there is an $f$-flex in the open interval between $p_{1}$ and $p_{n+1}$.

Remark. We do not assume any boundary condition in the proposition. This is possible since the points $p_{1}, \ldots, p_{n+1}$ are assumed to be interior points of $I$.

Proof. We may assume $n \geq 2$ since the case $n=1$ follows easily from the Kneser Lemma. We may also assume that $\left[p_{1}, p_{n+1}\right] \cap F\left(p_{1}, \ldots, p_{n}\right)$ consists of isolated points, since otherwise there is either an $f$-flex in $\left(p_{1}, p_{n+1}\right)$ or we can find a smaller interval 
with $n+1$ points from $F\left(p_{1}, \ldots, p_{n+1}\right)$ whose intersection with $F\left(p_{1}, \ldots, p_{n+1}\right)$ consists of isolated points.

Assume that $p_{1}$ occurs $j$ times in the sequence $p_{1}, \ldots, p_{n+1}$. We can assume that $F\left(p_{1}, \ldots, p_{n}\right) \cap\left(p_{1}, p_{j+1}\right)$ is empty. If $j=n$ the claim follows from Lemma 3.8. We therefore assume that $j<n$. Then we can consider the intrinsic system $g=f_{p_{n+1}, p_{n}, \ldots p_{j+3}, p_{j+2}}$ of order $2 j+1$ restricted to $\left[p_{1}, p_{j+1}\right]$ that we obtain by iterating the definition before Lemma 3.7. This intrinsic system satisfies the conditions in Lemma 3.8. There is therefore a $g$-flex $p_{1}^{\prime}$ in the open interval $\left(p_{1}, p_{j+1}\right)$. This implies that $f\left(p_{1}, \ldots, p_{n}\right)\left(p_{1}^{\prime}\right) \geq 2(j+1)$. We can therefore replace $p_{1}, \ldots, p_{j+1}$ by $p_{1}^{\prime}$ repeated $j+1$ times in the sequence $p_{1}, \ldots, p_{n+1}$. We can continue this argument inductively until we are in the situation that $p_{1}$ occurs $n$ times in the sequence $p_{1}, \ldots, p_{n+1}$ and we can use Lemma 3.8 to find the $f$-flex whose existence is claimed.

We now apply the methods of this section to prove a rather weak existence theorem for $f$-flexes of an intrinsic system defined on the whole circle.

3.11. Corollary. Let $f$ be an intrinsic system of order $2 n+1 \geq 5$ on $S^{1}$. Then $f$ has at least three $f$-flexes.

Remark. This assertion is optimal for $n=2$ as can be seen by either considering sextactic points, see [TU2], or periodic functions, see the example after Theorem 5.1. in Section five. We do not know whether it is optimal for $n>2$, but find it unlikely. In the special case of intrinsic systems of order $2 n+1$ coming from periodic functions we will prove in Section five the existence of at least $n+1$ points that are $f$-flexes, which is optimal.

Proof. We first prove the existence of two $f$-flexes. Let $p$ be some point on $S^{1}$ that is not an $f$-flex. If such $p$ does not exist there is nothing to prove. Notice that $p$ is isolated in $F\left(p^{n}\right)$. Let $p_{1}$ and $p_{2}$ be the next points to $p$ in $F\left(p^{n}\right)$ on each side of $p$. It could happen that $p_{1}$ and $p_{2}$ coincide. By Lemma 3.8 there is an $f$-flex in the open interval $\left(p_{1}, p\right)$ and another one in the open interval $\left(p, p_{2}\right)$.

Now we prove the existence of a third $f$-flex. Denote the $f$-flexes we have found by $q_{1}$ and $q_{2}$. We first consider the possibility that $F\left(q_{1}, q_{2}^{n-1}\right)$ only consists of $q_{1}$ and $q_{2}$. Then it follows from the Contraction Lemma 3.9 (or 3.10) that there is an $f$-flex in the open interval between $q_{1}$ and $q_{2}$ and another one in the open interval between $q_{2}$

and $q_{1}$. If $F\left(q_{1}, q_{2}^{n-1}\right)$ has a point $q_{3}$ that is different from $q_{1}$ and $q_{2}$, then we can after renaming $q_{1}$ and $q_{2}$ if necessary assume that $q_{3}$ lies in the open interval between $q_{1}$ and $q_{2}$. The Contraction Lemma 3.10 then implies the existence of an $f$-flex in the open interval between $q_{1}$ and $q_{2}$. This finishes the proof of the corollary.

\section{$\S 4$ The Jackson Lemmas}

We next prove two theorems - one for functions, the other for curves - which we will call Jackson Lemmas, since a similar result for vertices on simple closed arcs was found and applied by Jackson in [Ja], although the existence of vertices having inscribed or circumscribed osculating circles were not discussed in [Ja]. These two result will be used in Section five to prove the two theorems stated in the introduction. 
A piecewise $C^{2 n}$-function $u$ will be said to have a downward (resp. upward) singularity at a singular point $s$, if the interior angle in $s$ of the region above (resp. below) $u$ is less than or equal to $\pi$.

4.1 Theorem. (The Jackson Lemma for Flexes of Functions) Let $\mathcal{A}$ be a Chebyshev space of order $2 n+1$. Let $u$ be a piecewise $C^{2 n}$-function with at most one singularity which we then denote by a. Suppose $u \notin \mathcal{A}$ and that a is not an upward singular point of $u$. Then $u$ has at least one clean maximal $\mathcal{A}$-flex with the property that the osculating function there does not have the same value as $u$ in a.

We first prove the following weaker version of the Jackson Lemma.

4.2 Lemma. Let $\mathcal{A}$ and $u$ be as above. Then u has at least one global $\mathcal{A}$-flex s such that the osculating function $\varphi_{s}$ does not have the same value as $u$ in a and $\varphi_{s} \geq u$.

Remark. The flex whose existence is claimed in Lemma 4.2 is an $f_{u}$-flex with respect to the intrinsic system $f_{u}$ that can be associated to $u$ (on a sufficiently large closed interval $I$ not containing $a$ ).

Proof. We may assume that $a=0$. We fix $n$ mutually distinct points $p_{1} \prec \ldots \prec p_{n}$ arbitrarily, but all different from $a$. Since $u$ is not in $\mathcal{A}$, we can assume that none of the points $p_{1}, \ldots, p_{n}$ is a flex. We would like to show that the points $p_{1}, \ldots, p_{n}$ can be chosen such that $a \notin F_{u}\left(p_{1}, \ldots, p_{n}\right)$. Assume $a \in F_{u}\left(p_{1}, \ldots, p_{n}\right)$. We choose points $q_{1}, \ldots, q_{n}$ as follows:

$$
q_{1} \in\left(0, p_{1}\right), \quad q_{2} \in\left(p_{1}, p_{2}\right), \quad \ldots, \quad q_{n} \in\left(p_{n-1}, p_{n}\right) .
$$

Since $p_{1}, \ldots, p_{n}$ are not flexes, it follows that $F_{u}\left(p_{1}, \ldots, p_{n}\right)$ does not contain any of the intervals $\left(0, p_{1}\right), \ldots,\left(p_{n-1}, p_{n}\right)$ and hence also that we can choose $q_{1}, \ldots, q_{n}$ such that they are not contained in $F_{u}\left(p_{1}, \ldots, p_{n}\right)$. (See Figure 3. We indicate a periodic function $f(t)$ by the curve $\exp (u(t)-f(t))(\cos t, \sin t)$ on $\mathbf{R}$.) In particular, the function $f(t)=u(t)$ is expressed by the unit circle, and $f(t)=\varphi_{u}\left(p_{1}, \ldots, p_{n}\right)$ is expressed as a closed curve inscribed in the circle.

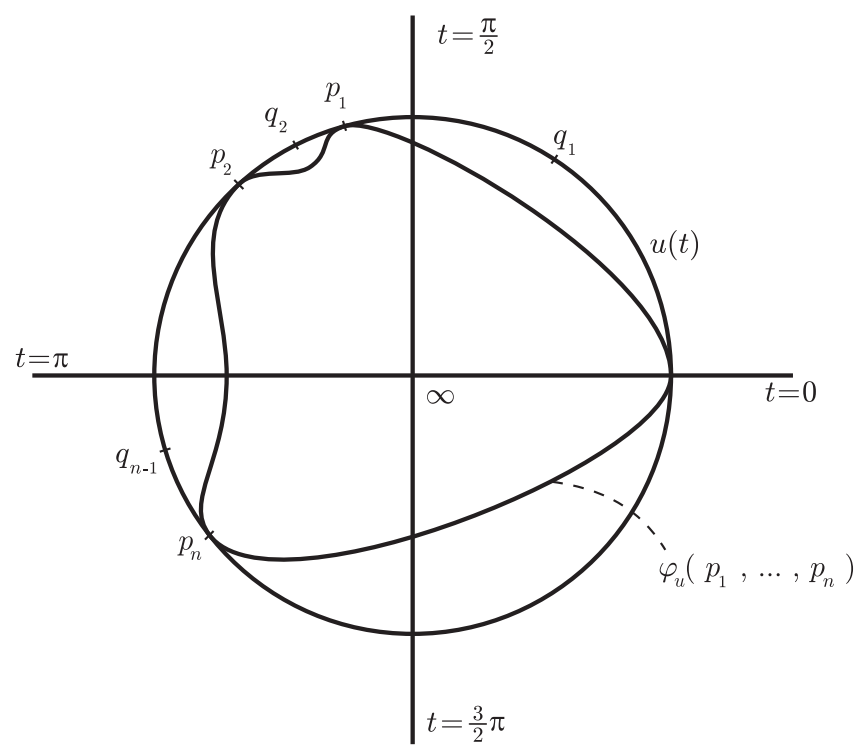

Figure 3. 
The graphs of $\varphi_{u}\left(p_{1}, \ldots, p_{n}\right)$ and $\varphi_{u}\left(q_{1}, \ldots, q_{n}\right)$ can at most meet in $2 n$ points counted with multiplicities since they are different. Hence they cannot meet in $a$. Now let $I$ be a closed interval not containing 0 , but containing $F_{u}\left(p_{1}, \ldots, p_{n}\right)$ in its interior. Since the total multiplicity of $F_{u}\left(p_{1}, \ldots, p_{n}\right)$ is at least $2 n+2$, the Contraction Lemma 3.10 applied to $f_{u}$ restricted to $I^{n}$ implies the existence of a global $\mathcal{A}$-flex $s$ on $I \subset(0,2 \pi)$. Notice that we may assume that the interval $[0, s]$ does not consist of $\mathcal{A}$-flexes. We next show that there is such a flex with the property that the osculating function does not have the same value as $u$ in $a$.

Suppose that the osculating function $\varphi_{u}\left(s^{n}\right)$ has the same value as $u$ in $a$. In this case, $u(t)$ must be $C^{1}$ at 0 . Furthermore $\varphi_{u}\left(s^{n}\right)\left(t_{0}\right)-u\left(t_{0}\right)>0$ holds for some $t_{0} \in(0, s)$ since the interval $[0, s]$ does not consist of $\mathcal{A}$-flexes.

We define a function $v$ as follows:

$$
v(t)= \begin{cases}u(t) & \text { if } t \in[0, s] \\ \varphi_{u}\left(s^{n}\right)(t) & \text { if } t \notin[0, s]\end{cases}
$$

Since $f_{v}$ is an intrinsic system of order $2 n+1$ on $[0, s]$ satisfying the boundary regularity condition $(1, n)$, we have by Lemma 3.7 that $g:=\left(f_{v}\right)_{s^{n-1}}$ is an intrinsic system of order 3 on $[0, s]$ satisfying

$$
g(0) \geq 2, \quad g(s) \geq 2
$$

Since $\varphi_{u}\left(s^{n}\right)\left(t_{0}\right)-u\left(t_{0}\right)>0$ holds for some $t_{0} \in(0, s)$, there is a point $r \in(0, s)$ such that $G(r)$ is connected and $G(r) \subset(0, s)$ by the Kneser Lemma 3.6. We define a new piecewise $C^{2 n}$-function on $S^{1}$ :

$$
w(t)= \begin{cases}u(t) & \text { if } t \in[r, s] \\ \varphi_{u}\left(r, s^{n-1}\right)(t) & \text { if } t \notin[r, s]\end{cases}
$$

Then we can define an intrinsic system $f_{w}$ of order $2 n+1$ satisfying the boundary regularity condition $(2, n-1)$ on $[r, s]$. Moreover we have

$$
f_{w}\left(r, s^{n-1}\right)(r) \geq 4, \quad f_{w}\left(r, s^{n-1}\right)(r) \geq 2(n-1) .
$$

Thus by the Contraction Lemma 3.9, we find a global $\mathcal{A}$-flex $s^{\prime}$ of $w$ on $(r, s)$, which is a global $\mathcal{A}$-flex of $u$. Since $G(r) \subset(0, s)$, we have $w(a)=\varphi_{u}\left(r, s^{n-1}\right)(a)>u(a)$. Thus the osculating function at $s^{\prime}$ does not have the same value as $u$ in $a$.

Proof of Theorem 4.1. We let $\Phi(u)$ denote the set of global $\mathcal{A}$-flexes of $u$ with the property that the osculating functions at the flexes do not have the same value as $u$ in $a$. By Lemma $4.2, \Phi(u)$ is nonempty. For $p \in \Phi(u)$ we let $I(p)$ denote the minimal closed interval in the complement of $a$ that contains $F_{u}\left(p^{n}\right)$. We define a new piecewise $C^{2 n}$-function $u_{p}$ on $S^{1}$ without upward singularities by setting (See Figure 4.)

$$
u_{p}(t)= \begin{cases}u(t) & \text { if } t \in I(p) \\ \varphi^{u}\left(p^{n}\right)(t) & \text { otherwise }\end{cases}
$$




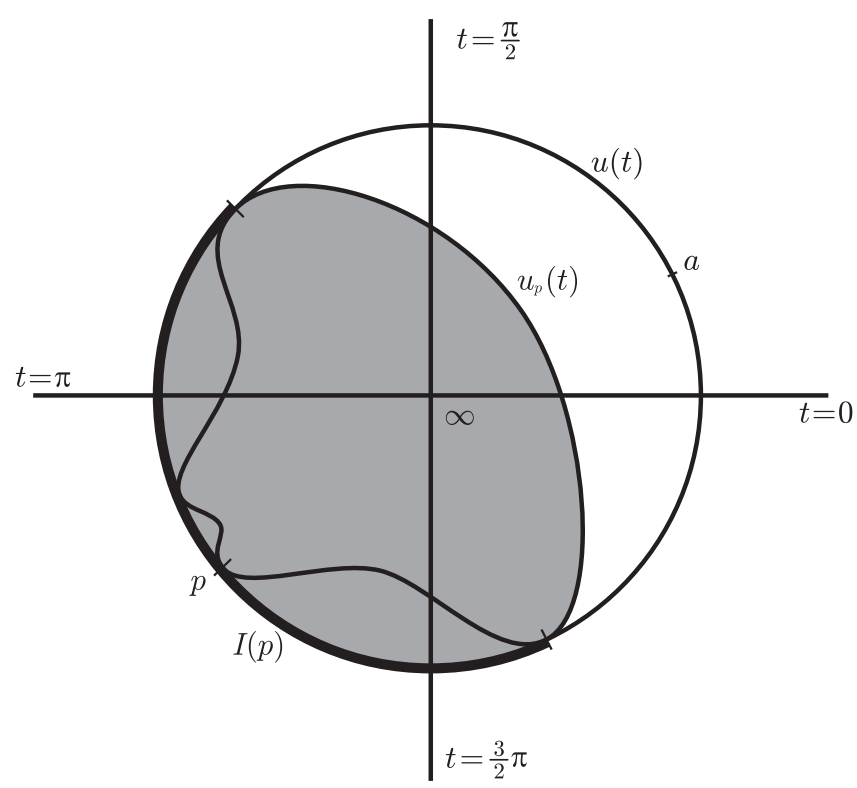

Figure 4.

We now define a partial ordering on $\Phi(u)$ by setting $p \ll q$ for $p, q \in \Phi(u)$ if

$$
I(p) \subset I(q) \quad \text { and } \quad u_{q} \leq u_{p}
$$

It is easy to check that this is in fact a partial ordering.

We next show that an element $p \in \Phi(u)$ that is minimal with respect to this partial ordering is a clean flex, or, in other words, $F_{u}\left(p^{n}\right)=I(p)$. Assume that such a minimal element $p$ is not a clean flex of $u$. Then $F_{u}\left(p^{n}\right)$ has at least two connected components. Then there is a point $q \in F_{u}\left(p^{n}\right)$ which belongs to a connected component of $F_{u}\left(p^{n}\right)$ not containing $p$. We can assume that the open interval bounded by $q$ and $p$ does not contain an $f_{u}$-flex. We consider the case $q \prec p$ (the case $p \prec q$ being similar). We consider the piecewise $C^{2 n}$-function $w$ on $S^{1}$ defined as

$$
w(t)= \begin{cases}u(t) & \text { if } t \in[q, p] \\ \varphi_{p}(t) & \text { if } t \notin[q, p]\end{cases}
$$

where $\varphi_{p}(t)$ is the osculating function of $u(t)$ at $p$. The function $w$ is a $C^{2 n}$-function on the interval $[q, p]$ satisfying the boundary regularity condition $(1, n)$, and we see from the Contraction Lemma 3.9 that there exists an $f_{w}$-flex $s$ in the open interval $(q, p)$. The osculating function $\varphi_{w, s}$ of $w$ at $s$ is equal to the osculating function $\varphi_{u, s}$ of $u$ at $s$ and

$$
\varphi_{u, s}=\varphi_{w, s} \geq w \geq u_{p} \geq u
$$

Thus $s$ is also an $f_{u}$-flex. Notice that $s \in \Phi(u)$, since $\varphi_{u, s}(a) \geq u_{p}(a)>u(a)$. Furthermore $s \ll p$ since $\varphi_{u, s}(t) \geq \varphi_{u, p}(t)$ for $t \notin I(p)$. Since $s \neq p$, this is a contradiction and we have proved that a point $p$ which is minimal with respect to the partial ordering is a clean flex.

We will now prove that there is a minimal point with respect to the partial ordering with help of Zorn's Lemma. Let $S$ be an arbitrary totally ordered subset of $\Phi(u)$. We fix 
some point $p_{0} \in S$ and let $S_{0}$ denote the set of elements $p \in S$ such that $p \ll p_{0}$. For $t \in S^{1} \backslash I\left(p_{0}\right)$, we set

$$
\varphi(t)=\sup \left\{\varphi_{u, p}(t) \mid p \in S_{0}\right\} .
$$

Notice that $\varphi_{u, p}$ depends continuously on $p$ since it is an osculating function of $u$ and $u$ is $C^{2 n}$. Hence the family of osculating functions of $u$ is bounded and the function $\varphi(t)$ is well defined. We would like to show that $\varphi(t)$ is the restriction to $S^{1} \backslash I\left(p_{0}\right)$ of a function in $\mathcal{A}$. We fix $2 n+1$ distinct points $t_{1}, \ldots, t_{2 n+1}$ on $S^{1}$ and set

$$
\alpha_{j}=\sup \left\{\varphi_{u, p}\left(t_{j}\right) \mid p \in S_{0}\right\} \quad \text { for } \quad j=1, \ldots, 2 n+1
$$

Then there exists a unique function $\psi(t) \in \mathcal{A}$ such that

$$
\psi\left(t_{j}\right)=\alpha_{j}
$$

There is a sequence $\left(p_{k}\right)$ in $S_{0}$ such that

$$
\alpha_{j}=\lim _{k \rightarrow \infty} \varphi_{u, p_{k}}\left(t_{j}\right) \quad \text { for } \quad j=1, \ldots, 2 n+1 .
$$

It follows that the sequence $\left(\varphi_{u, p_{k}}\right)$ converges uniformly to $\psi$ on $S^{1}$. Suppose that $\psi(c)<$ $\varphi(c)$ for some $c \in S^{1} \backslash I\left(p_{0}\right)$. Since $\varphi(c)=\sup \left\{\varphi_{u, p}(c) ; p \in S_{0}\right\}$, there exists $q \in S_{0}$ such that $\psi(c)<\varphi_{u, q}(c)$. In particular $\varphi_{u, p_{k}}(c)<\varphi(c)$. Since $S_{0}$ is a totally ordered set, we have $\varphi_{u, p_{k}}(t) \leq \varphi_{u, q}(t)$ for all $t \in S^{1} \backslash I\left(p_{0}\right)$. There is some $k_{0}$ such that $q<p_{k_{0}}$. Hence $\varphi_{u, p_{k_{0}}}(t) \geq \varphi_{u, q}(t)$ and it follows that $\varphi_{u, p_{k}}(t)=\varphi_{u, q}(t)$ for $k \geq k_{0}$, contradicting $\psi(c)<\varphi_{u, q}(c)$. It follows that $\varphi$ is a restriction of the function $\psi$ in $\mathcal{A}$ to $S^{1} \backslash I\left(p_{0}\right)$ as we wanted to show. We can assume that $\left(p_{k}\right)$ converges to a point $p_{\infty}$. It is clear that $p_{\infty}$ is a flex and that $\varphi(t)=\varphi_{u}\left(p_{\infty}\right)$. Then it follows that $q \in \Phi(u)$ and $p_{\infty} \ll p$ for all $p \in S$ since $\varphi_{u}\left(p_{\infty}\right)$ is strictly larger than $u$ outside of the interval $I$ defined by

$$
I=\cap_{p \in S} I(p) .
$$

We can therefore use Zorn's Lemma to find a minimal point with respect to the partial ordering thereby proving the existence of the clean flex with the desired properties.

The following theorem is the analogue of the Jackson Lemma for sextactic points. It will be used in section five to prove the theorem on sextactic points from the introduction.

4.3 Theorem. (The Jackson Lemma for Sextactic Points) Let $\gamma: S^{1} \rightarrow \mathbf{R}^{2}$ be a simple closed curve which is not a conic and is everywhere $C^{4}$-regular except maybe in a given point a where we assume that it is $C^{4}$-regular from both left and right. We assume furthermore that $\gamma$ is bounds a convex region and that it is strictly convex except maybe in the point $a$. Then there is a clean sextactic point $s$ on $(a, a+2 \pi)$ with the property that the osculating conic at $s$ is inscribed and does not meet $\gamma(a)$. If furthermore, $\gamma$ is at least $C^{1}$ in a, then $\gamma$ has a clean sextactic point $s$ with the property that the osculating conic in $s$ is circumscribed and does not meet $\gamma(a)$.

Proof. In the proof we will assume that the curve $\gamma$ lies in $P_{2}$, i.e., we compactify $\mathbf{R}^{2}$ by adding a line at infinity. It was explained in Example 2.6 (ii) how a regular strictly convex 
curve in the affine plane gives rise to an intrinsic system. Here the situation is somewhat different since we are allowing a singular point $a$.

Without loss of generality, we may set $a=0$. Take two distinct points $p_{1}, p_{2} \in(0,2 \pi)$ which are not sextactic points. Consider a maximal inscribed conic $\Gamma_{p_{1}, p_{2}}^{\bullet}$ (resp. a minimal circumscribed conic $\Gamma_{p_{1}, p_{2}}^{\circ}$ ) passing through $p_{1}$ and $p_{2}$. Suppose $\Gamma_{p_{1}, p_{2}}^{\bullet}$ passes through $a$. Then $\gamma$ must be $C^{1}$ at $a$. Choose $q_{1} \in\left(0, p_{1}\right)$ and $q_{2} \in\left(p_{1}, p_{2}\right)$ such that $\gamma\left(q_{1}\right)$ and $\gamma\left(q_{2}\right)$ do not lie on $\Gamma_{q_{1}, q_{2}}^{\bullet}$. Then as in the first paragraph of the proof of Lemma 4.2, one sees that the conic $\Gamma_{q_{1}, q_{2}}^{\bullet_{2}}$ does not pass through $\gamma(a)$. Hence we may assume that the conic conic $\Gamma_{p_{1}, p_{2}}^{\bullet}$ itself does not pass through $\gamma(a)$.

Let $I$ be a closed interval in $(0,2 \pi)$ such that $p_{1}, p_{2} \in I$. Then the function $f_{2}^{\bullet}$ defined in Example 2.6 (ii) is an intrinsic system system of order 5 on $I$ satisfying the boundary regularity condition $(5,5)$. We can prove the existence of a sextactic point on $(0,2 \pi)$ with the property that the osculating conic at $s$ is inscribed and does not meet $\gamma(a)$ with methods as in the proof of Lemma 4.2; see also Lemma 4.10 in [TU2] where this is also proved.

If furthermore $\gamma$ is $C^{1}$ at $a$, one can also assume that a minimal circumscribed conic $\Gamma_{p_{1}, p_{2}}^{\circ}$ does not pass through $\gamma(a)$ and show the existence of sextactic point on $(0,2 \pi)$ with the property that the osculating conic at $s$ is circumscribed and does not meet $\gamma(a)$. Here we use the fact that the function $f_{2}^{\circ}$ defined in Example 2.6 (ii) is an intrinsic system system of order 5 on $[\varepsilon, 2 \pi-\varepsilon]$ satisfying the boundary regularity condition $(5,5)$.

It is now straightforward how the arguments in the proof of the Jackson Lemma for Flexes of Functions 4.1 carry over to the present situation. We only sketch the main points. Let us assume that we are dealing with circumscribed conics assuming that $\gamma$ is $C^{1}$ at $a$. (The existence of a clean sextactic point whose osculating conic is inscribed is similar except that we do not need to assume $\gamma$ to be $C^{1}$ at $a$ since we have already proved the existence of sextactic point on $(0,2 \pi)$ with the property that the osculating conic at $s$ is circumscribed and does not meet $\gamma(a)$.)

Let $\Phi(\gamma)$ be the set of sextactic points such that the osculating function there are circumscribed and do not meet $a$. We have already seen that $\Phi(\gamma)$ is nonempty. For $p \in \Phi(\gamma)$ we define $I(p)$ to be the smallest closed interval containing $F_{2}^{\circ}\left(p^{2}\right)=\Gamma_{p^{2}}^{\circ} \cap \gamma$, but not containing $a$. Analogous to $u_{p}$ one defines $\gamma_{p}$ as $\gamma$ on the interval $I(p)$ and equal to $\Gamma_{p^{2}}^{\circ}$ on the complement of $I(p)$. Notice that $\gamma_{p}$ is a closed simple contractible curve in $P_{2}$. The partial ordering on $\Phi(\gamma)$ is now defined by setting $p \ll q$ for $p, q \in \Phi(u)$ if

$$
I(p) \subset I(q) \quad \text { and } \quad D\left(\gamma_{q}\right) \subset D\left(\gamma_{p}\right)
$$

where $D(c)$ denotes the closed contractible region bounded by a simple closed contractible curve $c$. The same arguments as in the proof of Theorem 4.1 can now be used to show that a point $p \in \Phi(\gamma)$ that is minimal with respect to this ordering is a clean sextactic point of the type we are are trying to find. The final step is again to use Zorn's Lemma to prove the existence of a minimal point in $\Phi(\gamma)$. Let $S$ be a totally ordered subset of $\Phi(\gamma)$. Set

$$
D=\bigcup_{p \in S} D\left(\gamma_{p}\right) .
$$


The boundary of $D$ consists of two pieces: One piece is the image of the interval

$$
I=\bigcap_{p \in S} I(p)
$$

under $\gamma$. We would like to show that the other piece, the complement of $\gamma(I)$ which we denote by $\Gamma$, is an arc of a conic. We choose five different points $q_{1}, \ldots, q_{5}$ on $\Gamma$ and five sequences $\left(q_{1, k}\right), \ldots,\left(q_{5, k}\right)$ of points on a sequence conics $\Gamma_{p_{k}^{2}}^{\circ}$ such that $\left(q_{i, k}\right)$ converges to $q_{i}$ for $i=1, \ldots, 5$. We can assume that the corresponding sequence $\left(p_{k}\right)$ converges to a point $p_{\infty}$. We can now use arguments as in the proof of Theorem 4.1 to show that arcs of $\Gamma_{p_{k}^{2}}^{\circ}$ converge to $\Gamma$ and that $\Gamma$ is an arc of the osculating conic $\Gamma_{p_{\infty}^{2}}^{\circ}$. It follows that $p_{\infty} \in \Phi(\gamma)$ and $p_{\infty} \ll p$ for all $p \in S$ and we can apply Zorn's Lemma.

\section{$\S 5$ On the existence of $2 n+2$ clean flexes on periodic functions}

We proved in Section three that a smooth periodic function $u$ has at least three flexes where the osculating functions are greater or equal to $u$ and similarly at least three flexes where the osculating functions are less than or equal to $u$. In Section four we also started to study the existence of clean flexes in the Jackson Lemma and this will be continued in this section.

Our main result here is the following theorem which is the same as Theorem 1.1 in the introduction if $\mathcal{A}=A_{2 n+1}$.

5.1. Theorem. Let $\mathcal{A}$ be a Chebyshev space of order $2 n+1$ where $n \geq 1$. Let $u$ be a $C^{2 n}$-function on $S^{1}$ which does not belongs to $\mathcal{A}$. Then $u(t)$ has at least $n+1$ different (intervals of) clean maximal $\mathcal{A}$-flexes and at least $n+1$ different (intervals of) clean minimal $\mathcal{A}$-flexes.

Remark. Notice that a clean maximal $\mathcal{A}$-flex cannot be a clean minimal $\mathcal{A}$-flex if $u$ does not belong to $\mathcal{A}$. The theorem therefore gives us $2 n+2$ clean flexes.

Example. Theorem 5.1 is optimal. Set $\mathcal{A}=A_{2 n+1}$. The flexes of a given function $u$ are the zeros of $L_{2 n+1} u$ where $L_{2 n+1}$ is the operator

$$
L_{2 n+1}=D\left(D^{2}-1\right) \cdots\left(D^{2}-n^{2}\right),
$$

where $D=d / d t$, see Proposition A.7 in Appendix A. If we set $u(t)=\sin (n+1) t$, then $L_{2 n+1} u(t)$ is proportional to $u(t)$. Thus $u(t)$ has exactly $2 n+2$-flexes which are all clean.

The main new tool in the proof of Theorem 5.1 is the following proposition, which is in the same spirit as the induction argument in [Ba], p. 201-204; see also Hilfssatz 2 on page 140 in [Nö]. Notice that unlike here, global properties are not treated in [Ba] and [Nö].

5.2. Proposition. Let $\mathcal{A}$ be a Chebyshev space of order $2 n+1$ where $n \geq 2$. Let $u$ be a $C^{2 n}$-function on $S^{1}$ and $f_{u}$ the corresponding intrinsic system of order $2 n+1$ on $S^{1}$.

(i) Suppose that

$$
f_{u}\left(p, a^{n-1}\right)(a) \geq 2 n
$$


and that $F_{u}\left(p, a^{n-1}\right) \backslash\{p\}$ is a closed interval where $p$ and a are two different points. Then both $(p, a)$ and $(a, p)$ contain a clean maximal $\mathcal{A}$-flex.

(ii) Let $[a, b]$ be a nontrivial closed interval on $S^{1}$. Suppose that

$$
f_{u}\left(p, a^{n-1}\right)(a) \geq 2 n \quad \text { and } \quad f_{u}\left(p, b^{n-1}\right)(b) \geq 2 n
$$

for a point $p \notin[a, b]$. If furthermore both $F_{u}\left(p, a^{n-1}\right) \backslash\{p\}$ and $F_{u}\left(p, b^{n-1}\right) \backslash\{p\}$ are different closed intervals, then there is a clean maximal $\mathcal{A}$-flex in the interval $(a, b)$.

Proof. We first proof the claim in (i). Instead of the original function $u$ we consider the following function (See Figure 5.)

$$
v(t)= \begin{cases}u(t) & \text { if } t \in[p, a] \\ \varphi_{u,\left(p, a^{n-1}\right)}(t)=\varphi_{u,\left(a^{n}\right)}(t) & \text { otherwise }\end{cases}
$$

where $\varphi_{u,\left(p, a^{n-1}\right)}$ is a minimal function with respect to $\left(p, a^{n-1}\right)$.

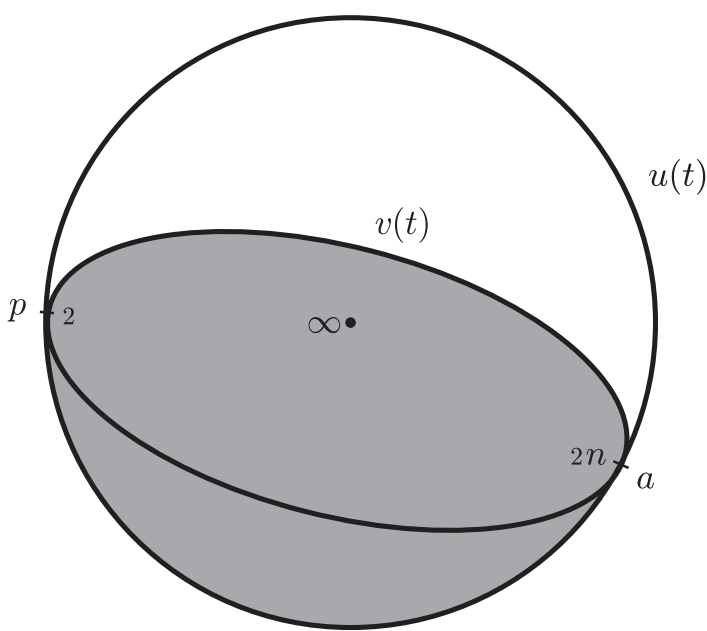

Figure 5 .

The function $v(t)$ is at least $C^{1}$ in $p$ and at least $C^{2 n-1}$ in $a$. Set $I=[p, a]$. Then $v$ satisfies the boundary condition $(1, n)$ on $I$; see Definition 2.2. We consider the intrinsic system $f_{v}$ defined on $I_{(1, n)}^{n}$. We have that

$$
f_{v}\left(p, a^{n-1}\right)(p)+f_{v}\left(p, a^{n-1}\right)(a) \geq 2(n+1)
$$

We can therefore apply the Contraction Lemma 3.9 to this situation which now implies that there is an $f_{v}$-flex $s$ in the open interval $(p, a)$ which is clearly also an $f_{u}$-flex. We have nothing to prove if $s$ is a clean flex of $u$. Therefore we assume that it is not. The osculating 
conic $\varphi_{u,\left(s^{n}\right)}$ can clearly only take on the same values as $u$ in the interval $[p, a] \cup F_{u}\left(p, a^{n-1}\right)$ since $v$ is strictly larger than $u$ on its complement. Let $q \in F_{u}\left(s^{n}\right)$ be point that is not in the same component of $F_{u}\left(s^{n}\right)$ as $s$. Let us assume that $q$ comes before $s$ in the interval $[p, a] \cup F_{u}\left(p, a^{n-1}\right)$, the other case being similar. Define a function $w$ by setting

$$
w(t)= \begin{cases}u(t) & \text { if } t \in[q, s] \\ \varphi_{u,\left(s^{n}\right)}(t) & \text { otherwise. }\end{cases}
$$

This function $w$ is $C^{2 n}$ in $s$ since $s$ is a flex. Hence $w$ is $C^{2 n}$ except possibly in $q$ where it is at least $C^{1}$. We can now apply the Jackson Lemma 4.1 to $w$. It follows that $w$ has a clean flex $s^{\prime}$ whose osculating function does not take on the same value as $u$ in $q$. Hence $s^{\prime}$ must be contained in the interval $(q, s)$. Notice also that $s^{\prime}$ cannot be contained in the interval $F_{u}\left(p, a^{n-1}\right) \backslash\{p\}$ since then $\varphi_{u,\left(s^{\prime}\right)}=\varphi_{u,\left(p, a^{n-1}\right)}$ contradicting that $s^{\prime}$ is a clean flex. It follows from this discussion that $s^{\prime} \in(p, a)$.

The proof that $(a, p)$ contains a clean flex is very similar.

Next we prove (ii). Instead of the original function $u(t)$, we consider the following function (See Figre 6.)

$$
v(t)= \begin{cases}\varphi_{u,\left(p, a^{n-1}\right)}(t)=\varphi_{u,\left(a^{n}\right)}(t) & \text { if } t \in[p, a] \\ u(t) & \text { if } t \in[a, b] \\ \varphi_{u,\left(p, b^{n-1}\right)}(t)=\varphi_{u,\left(a^{n}\right)}(t) & \text { if } t \in[b, p+2 \pi],\end{cases}
$$

where $\varphi_{u,\left(p, a^{n-1}\right)}\left(\operatorname{resp} . \varphi_{u,\left(p, b^{n-1}\right)}\right)$ are minimal function with respect to $\left(p, a^{n-1}\right)$ (resp. $\left.\left(p, b^{n-1}\right)\right)$.

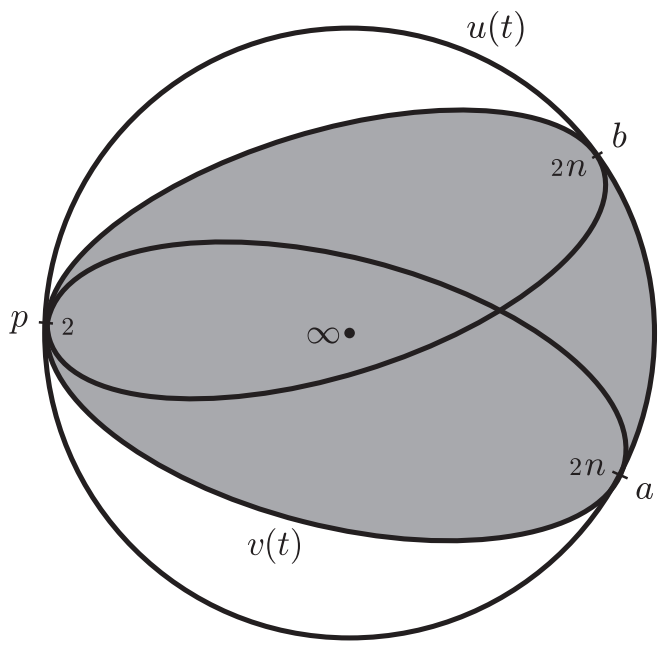

Figure 6.

The function $v(t)$ is at least $C^{1}$ in $p$ and $C^{2 n-1}$ in $a$ and $b$. Moreover $v$ satisfies at least the boundary regularity condition $(n, n)$ on $[a, b]$; see Definition 2.2. Let $f_{v}$ denote the 
intrinsic system of order $2 n+1$ on $[a, b]$ satisfying the boundary regularity condition $(n, n)$ whose existence was proved in Proposition 2.4. We choose $n$ different points $p_{1} \prec \cdots \prec p_{n}$ in the interval $(a, b)$, but not in $F_{u}\left(p, a^{n-1}\right) \cup F_{u}\left(p, b^{n-1}\right)$. Arguing exactly as at the beginning of the proof of the Jackson Lemma 4.1, we can assume that

$$
f_{v}\left(p_{1}, \cdots, p_{n}\right)(p)=0 \text {. }
$$

We choose a point $q \in\left(p, p_{n}\right)$ as follows: First we consider the case

$$
\sum_{i=1}^{n} f_{v}\left(p_{1}, \cdots, p_{n}\right)\left(p_{i}\right)=2 n .
$$

By Axiom (A6), there exists a point $q \in F_{v}\left(p_{1}, \cdots, p_{n}\right)$ that is different from $p$ and $p_{1}, \ldots, p_{n}$. Without loss of generality, we may assume that $q \in(p, b)$. After interchanging $q$ and $p_{1}, \ldots, p_{n}$ if necessary, we may assume

$$
q \prec p_{1} \prec \ldots \prec p_{n} .
$$

Next we consider the case that

$$
\sum_{i=1}^{n} f_{v}\left(p_{1}, \cdots, p_{n}\right)\left(p_{i}\right) \geq 2 n+2 .
$$

In this case we set $q=p_{1}$. In both of these two cases, there is a sufficiently small $\varepsilon>0$, such that $p+\varepsilon \prec q$ and $p_{n-1} \prec p_{n}-\varepsilon$. Moreover, $v$ is $C^{2 n-1}$ on $\left[p+\varepsilon, p_{n}-\varepsilon\right]$ satisfying at least the boundary regular condition $(n-1, n-1)$. By Proposition 2.8, we can associate to $v$ an intrinsic system $f_{v}^{\prime}$ of order $2 n-1$ on the interval $\left[p+\varepsilon, p_{n}-\varepsilon\right]$ with base point $p_{n}$. We have that

$$
\sum_{t \in\left[q, p_{n-1}\right]} f_{v}^{\prime}\left(p_{1}, \ldots, p_{n-1}\right)(t) \geq 2 n .
$$

Hence there exists a point $r \in\left(q, p_{n-1}\right)$ by the Contraction Lemma 3.10 such that

$$
f_{v}^{\prime}\left(r^{n-1}\right)(r) \geq 2 n
$$

If $r \notin[a, b)$, then $r \in(p, a)$. In this case $\varphi_{v,\left(p_{n}, r^{n-1}\right)}$ is locally around $r$ greater or equal to $\varphi_{u,\left(a^{n}\right)}$ and the two functions meet in $r$ with multiplicity at least $2 n$. Here $\varphi_{v,\left(p_{n}, r^{n-1}\right)}$ is the minimal function of $v$ with respect to $\left(p_{n}, r^{n-1}\right)$. On the other hand, the value of $\varphi_{v,\left(p_{n}, r^{n-1}\right)}$ in the point $p_{n}$ is smaller than that of $\varphi_{u,\left(a^{n}\right)}$ in $p_{n}$. This means that $\varphi_{v,\left(p_{n}, r^{n-1}\right)}$ meets $\varphi_{u,\left(a^{n}\right)}$ with multiplicity at least $2 n+2$ which implies that $\varphi_{v,\left(p_{n}, r^{n-1}\right)}=$ $\varphi_{u,\left(a^{n}\right)}$. Hence $p_{n} \in F_{u}\left(a^{n}\right)$ which is a contradiction. Thus we have $r \in[a, b)$.

We have

$$
f_{v}\left(r^{n-1}, p_{n}\right)(r)+f_{v}\left(r^{n-1}, p_{n}\right)\left(p_{n}\right) \geq 2(n+1) .
$$

Hence we can use the Contraction Lemma 3.9 (or 3.10) to prove the existence of a $f_{v}$-flex $s$ in the open interval $\left(a, p_{n}\right) \subset(a, b)$. The point $s$ is clearly also an $f_{u}$-flex, but might not be a clean flex. We can now as in the proof of part (i) of this proposition introduce a 
function $w$ and apply the Jackson Lemma to show the existence of a clean flex that must be contained in the interval $(a, b)$.

Proof of Theorem 5.1. We shall now prove the existence of $n+1$ clean maximal $\mathcal{A}$-flexes on $u$ by induction over the order $2 n+1$ of the Chebyshev space $\mathcal{A}$. The result can then be applied to $-u$ to also prove the existence of $n+1$ clean maximal $\mathcal{A}$-flexes on $u$.

If $n=1$, it follows quite easily from the proof of the Kneser Lemma 3.6 that $u$ has two clean maximal $\mathcal{A}$-flexes. We now assume the claim of the theorem to be true for $n-1$ and show that it then follows for $n$. We fix $p \in S^{1}$ that is not a flex. By Theorem A.2 in Appendix A there exists a function $\psi_{0} \in \mathcal{A}$ such that

$$
u(p)=\psi_{0}(p), \quad u^{\prime}(p)=\psi_{0}^{\prime}(p) .
$$

Now we define a linear subspace $V$ of $\mathcal{A}$ by setting

$$
V=\left\{\varphi \in \mathcal{A} ; \varphi(p)=0, \varphi^{\prime}(p)=0\right\} .
$$

We set

$$
\psi_{1}(t)=\alpha+\beta \cos t+\gamma \sin t
$$

We can adjust the three coefficients $\alpha, \beta, \gamma$ so that $\psi_{1}$ satisfies

$$
\psi_{1}(p)=\psi_{1}^{\prime}(p)=0 \text {. }
$$

Since $\psi_{1}$ belongs to $A_{3}$, it has at most two zeros counted with multiplicities. Thus $\psi_{1}$ has no zeros other than $p$ and the second derivative of $\psi_{1}$ at $p$ does not vanish. We then set

$$
\mathcal{A}_{\psi_{1}}=\left\{\frac{\varphi}{\psi_{1}} ; \varphi \in V\right\} .
$$

It can easily be checked that $\mathcal{A}_{\psi_{1}}$ is a Chebyshev space of order $2 n-1$ since $\varphi / \psi_{1}$ is $C^{2 n-2}$ at $p$; see Appendix B. We set

$$
v(t)=\frac{u-\psi_{0}}{\psi_{1}}
$$

Then $v$ is a $C^{2 n-2}$-function on $S^{1}$. So by applying the induction assumption there exist $n$ clean $\mathcal{A}_{\psi_{1}}$-flexes $s_{1}, \ldots, s_{n}$. Let $\varphi_{j}$ in $\mathcal{A}_{\psi_{1}}$ be the osculating function of $v$ at $s_{j}$. Since $\varphi_{j}$ is a function in $\mathcal{A}_{\psi_{1}}$, there exists a function $\hat{\varphi}_{j} \in V$ such that $\varphi_{j}=\hat{\varphi}_{j} / \psi_{1}$. If some $s_{j}$ is equal to $p$, then $\hat{\varphi}_{j}$ meets $u$ only in one component, and this implies that $p$ is a clean $\mathcal{A}$-flex, which is a contradiction.

So none of the points $s_{j}$ can be equal to $p$. Hence $\psi_{1}$ does not vanish in any of the points $s_{j}$ and it follows that the first $2 n-1$ derivatives of $\left(u-\psi_{0}\right)-\hat{\varphi}_{j}$ vanish in $s_{j}$. Since $\left(u-\psi_{0}\right)-\hat{\varphi}_{j}$ vanishes with multiplicity at least two in $p$, we have that

$$
\psi_{0}+\hat{\varphi}_{j}=\varphi_{\left(p, s_{j}^{n-1}\right)}=\varphi_{\left(s_{j}^{n}\right)}
$$

where $\varphi_{\left(p, s_{j}^{n-1}\right)}$ and $\varphi_{\left(s_{j}^{n}\right)}$ are the maximal functions of $u$ with respect to the $n$-uples $\left(p,\left(s_{j}\right)^{n-1}\right)$ and $\left(\left(s_{j}\right)^{n}\right)$ respectively. This implies that

$$
f_{u}\left(p, s_{j}^{n-1}\right)\left(s_{j}\right) \geq 2 n, \quad f_{u}\left(p, s_{j-1}^{n-1}\right)\left(s_{j-1}\right) \geq 2 n \quad \text { for } \quad j=0,1, \ldots, n, n+1,
$$


where we have set $s_{0}=s_{n+1}=p$. Since each $s_{j}$ is a clean $\mathcal{A}_{\psi_{1}}$-flex, $F_{u}\left(p, s_{j}^{n-1}\right) \backslash\{p\}$ is a closed interval for $j=1, \ldots, n$. By Proposition 5.2 , there is a clean maximal $\mathcal{A}$-flex $t_{j}$ on each of the intervals $\left(s_{j-1}, s_{j}\right)$ for $j=0, \ldots, n+1$.

We now also prove the other theorem stated in the introduction. The proof is similar to the one of Theorem 5.1.

5.3 Theorem. Let $\gamma: S^{1} \rightarrow \mathbf{R}^{2}$ be a strictly convex $C^{4}$-regular curve which is not a conic. Then $\gamma$ has at least three (intervals of) clean maximal and at least three (intervals of) clean minimal sextactic points.

Proof. We only prove the existence of three clean maximal sextactic points, the proof of the existence of three clean minimal flexes being similar. Let $f_{2}^{\bullet}$ be the intrinsic system of order 5 on $S^{1}$ introduced in Example 2.6 (ii). The following lemma is analogous to Proposition 5.2 (i) and has a very similar proof, which we therefore omit only remarking that it is this time based on the Jackson Lemma for Sextactic Points 4.3.

5.4. Lemma. Suppose that

$$
f_{2}^{\bullet}(p, a)(a) \geq 4
$$

and that $F^{\bullet}(p, a) \backslash\{p\}$ is a closed interval where $p$ and a are two different points. Then both $(p, a)$ and $(a, p)$ contain a clean maximal sextactic point.

We now come back to the proof of Theorem 5.3. By Theorem 4.3 there is a clean maximal sextactic point $p$ on the curve $\gamma$. We can also use Theorem 4.3 to find a clean maximal sextactic point $q$ whose osculating conic does not meet $p$. We first show that $f_{2}^{\bullet}(p, q)(p)=2$ and $f_{2}^{\bullet}(p, q)(q)=2$. Since the osculating conics coincide with the maximal inscribed conic at $p$ and $q$, the inequality $f_{2}^{\bullet}(p, q)(p) \geq 4\left(\right.$ or $\left.f_{2}^{\bullet}(p, q)(q) \geq 4\right)$ implies $f_{2}^{\bullet}(p, q)(p)=f_{2}^{\bullet}\left(p^{2}\right)(p)=\infty\left(\right.$ or $\left.f_{2}^{\bullet}(p, q)(q)=f_{2}^{\bullet}\left(q^{2}\right)(q)=\infty\right)$ and the osculating conic at $p$ would pass through $q$ (or the one at $q$ through $p$.) This is a contradiction. Hence $f_{2}^{\bullet}(p, q)(p)=f_{2}^{\bullet}(p, q)(q)=2$.

By (A6), there is a point $r$ distinct from $p, q$ such that $f^{\bullet}(p, q)(r) \geq 2$. Assume that $p \prec q \prec r$. The restriction of $f_{2}^{\bullet}$ to $[p, r]$ is an intrinsic system of order 5 satisfying the boundary regularity condition $(2,2)$. We can therefore define $f_{2, p}^{\bullet}$ as before Lemma 3.7., i.e., we set for $x \in[p, r]$ and $y \in S^{1}$

$$
f_{2, p}^{\bullet}(x)(y)= \begin{cases}f_{2}^{\bullet}(p, x)(y) & \text { if } y \neq p \\ f_{2}^{\bullet}(p, x)(y)-2 & \text { if } y=p\end{cases}
$$

By Lemma 3.7 we know that $f_{2, p}^{\bullet}$ restricted to $[q, r]$ is an intrinsic system of order 3 satisfying at least the boundary regularity condition $(1,1)$. There is now a subinterval $[a, b]$ of $[q, r]$ satisfying the conditions in the Kneser Lemma 3.6 which implies that there is a point $c$ in $(a, b)$ such that $F_{2, p}^{\bullet}(c)$ is connected. This implies that $f_{2}^{\bullet}(p, c)(c) \geq 4$ and that $F_{2}^{\bullet}(p, c) \backslash\{p\}$ is a closed interval. By Lemma 5.4 we then have a clean maximal point in the interval $(p, c)$ and another one in $(c, p)$. (One of these sextactic points might coincide with q.) Since $p$ is also a clean sextactic point we have proved the existence of thee (intervals of) clean maximal sextactic points. 


\section{$\S 6$ Arrangements of clean flexes.}

As was pointed out in Example 2.6 (i), there are two clean maximal vertices and two clean minimal vertices on a given simple closed curve $\gamma: S^{1} \rightarrow \mathbf{R}^{2}$. It is a natural question to ask in which order the clean maximal and the clean minimal vertices are arranged on $S^{1}$. In [TU2], the authors proved that there are four points $t_{1} \prec t_{2} \prec t_{3} \prec t_{4}$ on $S^{1}$ such that $t_{1}, t_{3}$ are clean maximal vertices and $t_{2}, t_{4}$ are clean minimal vertices. Now that we have proved the existence of $2 n+2$ clean $\mathcal{A}$-flexes on a $2 \pi$-periodic function $u$, we can ask again how the maximal and minimal ones are arranged relative to each other. We will say that the clean $\mathcal{A}$-flexes on $u$ change sign at least $m$-times if there are $2 m$-points

$$
p_{1} \prec q_{1} \prec \cdots \prec p_{m} \prec q_{m}
$$

on $[0,2 \pi)$ such that $p_{j}$ for $j=1, \ldots, m$ are clean maximal $\mathcal{A}$-flexes and $q_{j}$ for $j=1, \ldots, m$ are clean minimal $\mathcal{A}$-flexes.

6.1 Theorem. Let $u$ be a $2 \pi$-periodic $C^{2 n}$-function which is not in $\mathcal{A}$. Then the clean $\mathcal{A}$-flexes on $u$ change sign at least four times.

We do not know whether Theorem 6.1 gives an optimal lower bound on the number of sign changes or not.

To prove the theorem, we will use the abstract theory of pairs of intrinsic circle systems of which we give a quick review.

6.2 Definition. A family of nonempty closed subsets $F=\left(F_{p}\right)_{p \in S^{1}}$ of $S^{1}$ is called an intrinsic circle system on $S^{1}$ if it satisfies the following three conditions for any $p \in S^{1}$.

(I1) If $q \in F_{p}$, then $F_{p}=F_{q}$.

(I2) If $p^{\prime} \in F_{p}, q^{\prime} \in F_{q}$ and $q \succeq p^{\prime} \succeq q^{\prime} \succeq p(\succeq q)$, then $F_{p}=F_{q}$ holds.

(I3) Let $\left(p_{n}\right)_{n \in \mathbf{N}}$ and $\left(q_{n}\right)_{n \in \mathbf{N}}$ be two sequences in $S^{1}$ such that $\lim _{n \rightarrow \infty} p_{n}=p$ and $\lim _{n \rightarrow \infty} q_{n}=q$ respectively. Suppose that $q_{n} \in F_{p_{n}}$ for all $n \in \mathbf{N}$. Then $q \in F_{p}$ holds.

A pair of intrinsic circle systems $\left(F^{+}, F^{-}\right)$is said to be compatible if it satisfies the following two conditions.

(C1) $F_{p}^{+} \cap F_{p}^{-}=\{p\}$ for all $p \in S^{1}$.

(C2) Suppose that $F^{+}(p)$ (resp. $F^{-}(p)$ ) is connected. Then there are no points $q$ in a sufficiently small neighborhood of $p$ such that $F^{+}(q)$ (resp. $F^{-}(q)$ ) is connected.

In [TU2], Theorem 1.4, the authors proved the following

6.3 Lemma. Let $\left(F^{+}, F^{-}\right)$be a compatible pair of intrinsic circle systems. Then there are four points $p_{1}, p_{2}, p_{3}, p_{4} \in S^{1}$ satisfying $p_{1} \succ p_{2} \succ p_{3} \succ p_{4}\left(\succ p_{1}\right)$ such that $F_{p_{1}}^{+}, F_{p_{3}}^{+}$ and $F_{p_{2}}^{-}, F_{p_{4}}^{-}$are connected subsets of $S^{1}$.

We now prove the theorem as a corollary of Lemma 6.3.

Proof of Theorem 6.1. Suppose that the claim in the theorem is not true. Then the clean flexes on $u$ change sign exactly two times. There are clearly disjoint closed intervals $I$ and $J$ containing all the clean maximal and all the clean minimal flexes respectively. There is a point $p \in S^{1}$ such that $p \notin I \cup J$. Without loss of generality, we may set $p=0$, and

$$
0<\inf (I)<\sup (I)<\inf (J)<\sup (J)<2 \pi
$$


We set

$$
\begin{aligned}
& F_{q}^{+}= \begin{cases}F_{u}\left(q, p^{n-1}\right) \backslash\{p\} & \text { if } q \notin F\left(p^{n}\right) \\
F_{u}\left(p^{n}\right) & \text { if } q \in F\left(p^{n}\right),\end{cases} \\
& F_{q}^{-}= \begin{cases}F_{-u}\left(q, p^{n-1}\right) \backslash\{p\} & \text { if } q \notin F\left(p^{n}\right) \\
F_{-u}\left(p^{n}\right) & \text { if } q \in F\left(p^{n}\right) .\end{cases}
\end{aligned}
$$

It is easy to check that $\left(F^{+}, F^{-}\right)$is a compatible pair of intrinsic circle systems. By Lemma 6.3 , there are four points $p_{1}, p_{2}, p_{3}, p_{4} \in S^{1}$ satisfying $p_{1} \succ p_{2} \succ p_{3} \succ p_{4}\left(\succ p_{1}\right)$ such that $F_{p_{1}}^{+}, F_{p_{3}}^{+}$and $F_{p_{2}}^{-}, F_{p_{4}}^{-}$are all connected. Now we claim that $p_{1}, p_{3} \in I$. Assume that $p_{1} \notin I$ holds, then we have $p_{1} \in(0, \inf (I))$ or $p_{1} \in(\sup (I), 2 \pi)$. Since $F_{p_{1}}^{+}$is connected, we have

$$
f_{u}\left(p_{1}, p^{n-1}\right)\left(p_{1}\right) \geq 4, \quad f_{u}\left(p_{1}, p^{n-1}\right)(p) \geq 2 n-2
$$

By Lemma 3.7, we can define a new intrinsic system $g$ of order $2 n-2$ on $\left[0, p_{1}\right]$ (resp. on $\left.\left[p_{1}, 2 \pi\right]\right)$ by

$$
g\left(q_{1}, \ldots, q_{n-1}\right)(r)= \begin{cases}f\left(p_{1}, q_{1}, \ldots, q_{n-1}\right)(r) & \text { if } q \neq r \\ f\left(p_{1}, q_{1}, \ldots, q_{n-1}\right)(r)-2 & \text { if } r=q\end{cases}
$$

where $\left(q_{1}, \ldots, q_{n-1}\right) \in\left[0, p_{1}\right]_{(1, n)}^{n-1}\left(\operatorname{resp} .\left(q_{1}, \ldots, q_{n-1}\right) \in\left[p_{1}, 2 \pi\right]_{(1, n)}^{n-1}\right)$. Since $g$ satisfies the boundary regularity condition $(1, n)$ on $\left[0, p_{1}\right]$ and $(n, 1)$ on $\left[p_{1}, 2 \pi\right]$, there are clean $g$ flexes $s_{1} \in\left[0, p_{1}\right]$ and $s_{2} \in\left[p_{1}, 2 \pi\right]$. (Apply Proposition 5.2 (i) to the Chebyshev space $A_{\psi_{1}}$ defined in the proof of Theorem 5.3.) Thus we have

$$
f\left(p_{1}, s_{j}^{n-1}\right)\left(p_{1}\right) \geq 2, \quad f\left(p_{1}, s_{j}^{n-1}\right)\left(s_{j}\right) \geq 2 n \quad(j=1,2) .
$$

By Proposition $5.2(\mathrm{i})$, we have a clean maximal $\mathcal{A}$-flex $s_{1}^{\prime} \in\left(0, s_{1}\right)$ and $s_{2}^{\prime} \in\left(s_{2}, 2 \pi\right)$ respectively. If $p_{1} \in(0, \inf (I))$ (resp. $\left.p_{1} \in(\sup (I), 2 \pi)\right)$, we have $s_{1}^{\prime} \in(0, \inf (I))$ (resp. $\left.s_{2}^{\prime} \in(\sup (I), 2 \pi)\right)$. This is a contradiction since all of the clean maximal flexes are contained in $I$. So we have $p_{1} \in I$. Similarly we can see that $p_{3} \in I$ and $p_{2}, p_{4} \in J$. This contradicts the relation $p_{1} \succ p_{2} \succ p_{3} \succ p_{4}$. Hence we must have at least four sign changes of clean flexes.

Finally we formulate two open problems which are in our opinion the most important ones on flexes of periodic functions.

Problems. (1) Give a best lower bounds for the number of sign changes of clean $\mathcal{A}$-flexes of a periodic function. The number $2 n+2$ is a tempting guess.

(2) Let $u$ be a $2 \pi$-periodic $C^{\infty}$-function and $\mathcal{S}$ be the union over the sets of $A_{2 n+1^{-}}$ flexes on $u$ where $n$ ranges over all natural numbers. Is the set $\mathcal{S}$ a dense subset of $S^{1}$ ?

\section{Appendix A. Chebyshev spaces.}

In this appendix, we shall define Chebyshev spaces as certain linear subspaces of smooth functions, bring their basic theory and explain the existence of $2 n+2$ flexes on a periodic function as an application.

Chebyshev spaces are related to Fourier series and disconjugate operators. It is well known that many theorems in the spirit of the classical four vertex theorem can be proved 
using either Fourier series (see Hayashi [Hy] and Blaschke [Bl], p. 68) or disconjugate operators (see [Ar2], [GMO], [OT] and [Ta]).

A $C^{n}$-function $u$ defined on $\mathbf{R}$ has a zero of order $m$ (or a zero with multiplicity $m$ ) at $s$ where $1 \leq m \leq n$ if the value and the first $m-1$ derivatives of $u$ at $s$ vanish, but not the $m$-th. Notice that we do not define the order of a zero $s$ of a $C^{n}$-function $u$ that vanishes in $s$ together with all its $n$ derivatives since it will not be needed in the following.

A.1 Definition. A linear subspace $\mathcal{A}$ of $C^{n-1}(\mathbf{R})$ is called a Chebyshev space of order $n$ if the following conditions are satisfied.

(i) $n \leq \operatorname{dim} \mathcal{A}$.

(ii) Every nonvanishing function $\varphi$ in $\mathcal{A}$ has at most $n-1$ zeros counted with multiplicity in $\mathbf{R} / 2 \pi \mathbf{Z}$.

(iii) Every function $\varphi$ in $\mathcal{A}$ is $2 \pi$-periodic if $n$ is odd.

(ii) Every function $\varphi$ in $\mathcal{A}$ is $2 \pi$-antiperiodic, i.e., $\varphi(t+2 \pi)=-\varphi(t)$, if $n$ is even.

In the definition of a Chebyshev space we allow the possibility that $\operatorname{dim} \mathcal{A}=\infty$. We will see in Theorem A.2 that the dimension of a Chebyshev space is always finite and equal to its order $n$.

We now give a few examples of Chebyshev spaces.

The space

$$
A_{2 k+1}=\left\{\varphi \in C^{2 k}(\mathbf{R}) ; \varphi(t)=a_{0}+\sum_{n=1}^{k}\left(a_{n} \cos n t+b_{n} \sin n t\right)\right\} .
$$

is a Chebyshev space of order $2 k+1$. In fact, we have

$$
\cos n t=\frac{z^{n}+z^{-n}}{2}, \quad \sin n t=\frac{z^{n}-z^{-n}}{2 i}
$$

where $z=e^{i t}$. The functions $z^{k} \varphi$ for $\varphi$ in $A_{2 k+1}$ are polynomials in $z$ of degree less than or equal to $2 k$. Consequently, the number of zeros of the functions $\varphi$ in $A_{2 k+1}$ can at most be $2 k$.

Similarly, the space

$$
A_{2 k}=\left\{\varphi \in C^{2 k}(\mathbf{R}) ; \varphi(t)=\sum_{n=1}^{k}\left[a_{n} \cos \left(\frac{(2 n-1) t}{2}\right)+b_{n} \sin \left(\frac{(2 n-1) t}{2}\right)\right]\right\} .
$$

is a Chebyshev space of order $2 k$.

A linear differential operator $L$ of order $n$ on $\mathbf{R}$ is called disconjugate if its kernel $\operatorname{Ker} L$ is a Chebyshev space of order $n$. Examples of disconjugate operators are

$$
\begin{aligned}
L_{2 k+1} & =D\left(D^{2}-1\right) \cdots\left(D^{2}-k^{2}\right), \\
L_{2 k} & =\left(D^{2}-\left(\frac{1}{2}\right)^{2}\right) \cdots\left(D^{2}-\left(\frac{2 k-1}{2}\right)^{2}\right),
\end{aligned}
$$

where $D=d / d t$, since their kernels are $A_{2 k+1}$ and $A_{2 k}$ respectively. We refer to Proposition A.6 for more information on Chebyshev spaces and disconjugate operators. 
Further simple examples of Chebyshev spaces can be obtained as follows. Let $\mathcal{A}$ be the linear span of either one of the sets

$$
\begin{aligned}
A_{2 k+1}^{n} & =\left\{\varphi_{1} \cdots \varphi_{n} ; \varphi_{1}, \ldots, \varphi_{n} \in A_{2 k+1}\right\}, \\
A_{2 k}^{n} & =\left\{\psi_{1} \cdots \psi_{n} ; \psi_{1}, \ldots, \psi_{n} \in A_{2 k}\right\} .
\end{aligned}
$$

Then $\mathcal{A}$ is a Chebyshev space of order $n(2 k+1)$ in the first case and of order $2 n k$ in the second case.

The following property of Chebyshev spaces is crucial.

A.2 Theorem. Let $\mathcal{A}$ be a Chebyshev space in $C^{n-1}(\mathbf{R})$ of order $n$. Let

$$
0 \leq t_{1} \leq t_{2} \leq \cdots \leq t_{n}<2 \pi
$$

be $n$ points and $\nu_{j}$ the multiplicity with which $t_{j}$ occurs as a component of the $(j-1)$-uple $\left(t_{1}, \ldots, t_{j-1}\right)$. Then the linear map $T: \mathcal{A} \rightarrow \mathbf{R}^{n}$ defined by

$$
T(\varphi)=\left(\varphi^{\left(\nu_{1}\right)}\left(t_{1}\right), \ldots, \varphi^{\left(\nu_{n}\right)}\left(t_{n}\right)\right)
$$

is an isomorphism. In particular, $\mathcal{A}$ is finite dimensional and its dimension coincides with the order $n$.

Proof. Let $\varphi \in \mathcal{A}$ be in the kernel of the map $T$. It follows from the definition of $T$ that $\varphi$ vanishes at least $n$ times counted with multiplicities. The definition of a Chebyshev space now implies that $\varphi$ vanishes identically. We have therefore proved that $T$ is injective and it follows that $\operatorname{dim} \mathcal{A} \leq n$. By the definition of a Chebyshev space we have $\operatorname{dim} \mathcal{A} \geq n$. Hence $\operatorname{dim} \mathcal{A}=n$ and $T$ is an isomorphism.

A.3 Corollary. Let $\mathcal{A}$ be a Chebyshev space of order $n$. Let

$$
0 \leq t_{1}<t_{2}<\cdots<t_{k}<2 \pi
$$

be $k$ different points where $k \leq n-1$ and let $i_{1}, i_{2}, \ldots, i_{k}$ be $k$ positive integers satisfying $i_{1}+i_{2}+\cdots+i_{k}=n-\ell$ for $\ell \geq 0$. Then the set of functions $\varphi$ in $\mathcal{A}$ which have zeros of order $i_{j}$ at $t_{j}$ for $j=1, \ldots, k$ is an $\ell$-dimensional subspace of $\mathcal{A}$.

We shall now prove the following

A.4 Theorem. Let $\mathcal{A}$ be a Chebyshev space in $C^{n-1}(\mathbf{R})$ of odd (resp. even) order $n$. Let $u$ be a nonvanishing $2 \pi$-periodic (resp. $2 \pi$-antiperiodic) $C^{n-1}$-function. Suppose

$$
\int_{S^{1}} u(t) \varphi(t) d t=0
$$

for all $\varphi(t) \in \mathcal{A}$. Then the function $u(t)$ changes its sign at least $n+1$ times on $S^{1}$.

Proof. Suppose that $u$ does not change sign if $n$ is odd and that it changes sign only ones if $n$ is even. Let $t_{1}$ be arbitrary if $n$ is odd and the zero of $u$ if $n$ is even. By Corollary A.3 there is a nonvanishing function $\varphi$ in $\mathcal{A}$ with a zero in $t_{1}$ with multiplicity $n-1$. We can 
choose the sign of $\varphi$ such that $u(t) \varphi(t) \geq 0$ for all $t$. Then the integral in (3) being equal to zero implies that $u \varphi$ vanishes identically. This is a contradiction since both $u$ and $\varphi$ vanish in at most one point. Hence $u$ changes sign at least ones if $n$ is odd and at least twice if $n$ is even.

Now assume that $u$ changes sign only in the $m$ distinct points

$$
t_{1}<t_{2}<\cdots<t_{m}
$$

where $m<n+1$. Notice that $m$ is even if $n$ is odd and $m$ is odd if $n$ is even. Hence $n-m$ is an odd integer. By Corollary A.3, there exists a function $\psi$ in $\mathcal{A}$ such that

(i) $\psi$ has zero in $t_{1}$ with multiplicity $n-m$,

(ii) $\psi$ has a zero in $t_{j}$ with multiplicity one if $j \geq 2$.

Since the total multiplicity of zeros of $\psi$ is $n-1$, it follows that $\psi$ has no zeros other than $t_{1}, \ldots, t_{m}$. Since the multiplicities of the zeros at $t_{1}, \ldots, t_{m}$ are all odd integers, we have that $\psi$ changes its sign exactly at $t_{1}, \ldots, t_{m}$. Thus $u \psi$ never changes sign. The vanishing of the integral in (3), now implies that $u$ must vanish identically, which is a contradiction. Hence $u$ changes it sign at least $n+1$ times.

Let $\mathcal{A}$ be a Chebyshev space of odd (resp. even) order $n$ in $C^{n-1}(\mathbf{R})$. Let $u$ be a nonvanishing $2 \pi$-periodic (resp. anti $2 \pi$-periodic) $C^{n-1}$-function. Let $s \in S^{1}$. A function $\varphi_{s} \in \mathcal{A}$ is called the $\mathcal{A}$-osculating function of $u$ at $s$ if the first $n-1$ derivatives of $\varphi_{s}$ and $u$ at $s$ coincide. That $\varphi_{s}$ exists and is unique follows from the bijectivity of the mapping $T$ in Theorem A.2 by setting $t_{1}=\cdots=t_{n}=s$.

A.5 Definition. Suppose $\mathcal{A}$ and $u$ as above are contained in $C^{n}(\mathbf{R})$. Then a point $s \in S^{1}$ is called an $\mathcal{A}$-flex if the $n$-th derivative of $\varphi_{s}$ and $u$ coincide in $s$.

¿From now on we assume that a Chebyshev space $\mathcal{A}$ of order $n$ is contained in $C^{n}(\mathbf{R})$. We will show in Theorem A.8 that the number of $\mathcal{A}$-flexes on an $2 \pi$-periodic (resp. $2 \pi$ antiperiodic) function $u$ in $C^{n}(\mathbf{R})$ is at least $n+1$. For this we will need the next proposition which is interesting in its own right. It shows that the condition that a Chebyshev space $\mathcal{A}$ of order $n$ be contained in $C^{n}(\mathbf{R})$ is a necessary and sufficient condition that it is the kernel of a disconjugate operator of order $n$.

Notice though that the concept of a Chebyshev space is essentially wider than that of kernels of disconjugate operators. An example of a Chebyshev space of order 3 which is in $C^{2}(\mathbf{R})$ but not in $C^{3}(\mathbf{R})$ can be obtained as follows. Let $\mathcal{A}_{\gamma}$ be the linear span of the functions $\{1, x, y\}$ where $\gamma=(x, y): S^{1} \rightarrow \mathbf{R}^{2}$ is a strictly convex $C^{2}$-curve which is not $C^{3}$. Clearly, $\mathcal{A}_{\gamma}$ is a Chebyshev space of order three since any line meets the curve $\gamma$ in at most two points counted with multiplicities.

A.6 Proposition. Let $\mathcal{A}$ be a Chebyshev space of odd (resp. even) order $n$ in $C^{n+m}(\mathbf{R})$ for $m \geq 0$, then there exists a unique differential operator of the form

$$
L_{\mathcal{A}}=D^{n}+a_{n-1} D^{n-1}+\cdots+a_{1} D+a_{0}
$$

such that $\mathcal{A}$ is the kernel of $L_{\mathcal{A}}$, where the coefficients $a_{j}$ are $2 \pi$-periodic (resp. $2 \pi$ antiperiodic) $C^{m}$-functions. 
The operator $L_{\mathcal{A}}$ in the proposition is called the disconjugate operator associated with the Chebyshev space $\mathcal{A}$. The uniqueness of $L_{\mathcal{A}}$ is due to the fact that its highest order coefficient is normalized to be 1 .

Proof. We fix a point $t \in S^{1}$ arbitrarily and define a linear map $T_{t}: \mathcal{A} \rightarrow \mathbf{R}^{n}$ by setting

$$
T_{t}(\varphi)=\left(\varphi(t), \varphi^{\prime}(t), \ldots, \varphi^{(n-1)}(t)\right) .
$$

By Theorem A.2, the map $T_{t}$ is bijective. We define a linear functional $S_{t}: \mathcal{A} \rightarrow \mathbf{R}$ by setting $S_{t}(\varphi)=\varphi^{(n)}(t)$. Since $S_{t} \circ T_{t}^{-1}$ is a linear functional on $\mathbf{R}^{n}$, there exists an $\left(a_{0}(t), \ldots, a_{n-1}(t)\right)$ in $\mathbf{R}^{n}$ such that

$$
S_{t} \circ T_{t}^{-1}\left(x_{0}, \ldots, x_{n-1}\right)=-\sum_{i=0}^{n-1} a_{i}(t) x_{i},
$$

where the choice of the negative sign in front of the sum will soon become clear. It is clear that $\left(a_{0}(t), \ldots, a_{n-1}(t)\right)$ is $C^{m}$ in $t$. Now we have

$$
\begin{aligned}
\varphi^{(n)}(t) & =S_{t}(\varphi) \\
& =S_{t} \circ T_{t}^{-1}\left(T_{t}(\varphi)\right) \\
& =S_{t} \circ T_{t}^{-1}\left(\varphi(t), \varphi^{\prime}(t), \ldots, \varphi^{(n-1)}(t)\right) \\
& =-\sum_{i=0}^{n-1} a_{i}(t) \varphi^{(i)}(t) .
\end{aligned}
$$

This proves the existence of the operator $L_{\mathcal{A}}$. The uniqueness is clear.

The next proposition is a further preparation for the existence of flexes in Theorem A.8.

A.7 Proposition Let $\mathcal{A}$ be a Chebyshev space of odd (resp. even) order $n$ in $C^{n}(\mathbf{R})$. Let $u$ be a $2 \pi$-periodic (resp. $2 \pi$-antiperiodic) $C^{n}$-function. A point $s$ is an $\mathcal{A}$-flex of $u$ if and only if the function $L_{\mathcal{A}} u$ vanishes at $s$.

Proof. Since $\mathcal{A}$ is contained in $C^{n}(\mathbf{R})$ it has an associated disconjugate operator $\mathcal{A}$ such that $L_{\mathcal{A}} \varphi=0$ for all $\varphi$ in $\mathcal{A}$. Let $\varphi_{s}$ in $\mathcal{A}$ be the osculating function of $u$ at a point $s$. Since $L_{\mathcal{A}}\left(\varphi_{p}\right)$ vanishes identically, we have that

$$
\begin{aligned}
u^{(n)}(t)-\varphi_{s}^{(n)}(t) & =u^{(n)}(t)+\sum_{i=1}^{n-1} a_{i}(t) \varphi_{s}^{(i)}(t) \\
& =u^{(n)}(t)+\sum_{i=0}^{n-1} a_{i}(t) u^{(i)}(t) \\
& =\left(L_{\mathcal{A}} u\right)(t)
\end{aligned}
$$

for all $t$. Hence $s$ is an $\mathcal{A}$-flex if and only if $L_{\mathcal{A}} u$ vanishes in $s$. 
A.8 Theorem Let $\mathcal{A}$ be a Chebyshev space of odd (resp. even) order $n$ in $C^{n}(\mathbf{R})$. Let $u$ be a $2 \pi$-periodic (resp. $2 \pi$-antiperiodic) $C^{n}$-function. Then the number of $\mathcal{A}$-flexes of $u$ on $S^{1}$ is at least $n+1$.

Proof. The adjoint operator $L^{*}$ of a disconjugate operator $L$ is also disconjugate; see Theorem 9 on p. 104 in [Co]. Let $\mathcal{A}^{*}$ be the Chebyshev space of order $n$ corresponding to $L_{\mathcal{A}}^{*}$ which is also contained in $C^{n}(\mathbf{R})$. Then

$$
\int_{S^{1}}\left(L_{\mathcal{A}} u\right)(t) \varphi(t) d t=\int_{S^{1}} u(t)\left(L_{\mathcal{A}}^{*} \varphi\right)(t) d t=0
$$

for all $\varphi$ in $\mathcal{A}^{*}$. We now apply Theorem A.4 to the Chebyshev space $\mathcal{A}^{*}$ and conclude that the function $L_{\mathcal{A}} u$ changes sign at least $n+1$ times. Hence $u$ has at least $n+1$ flexes by Proposition A.7.

Theorem A.8 is optimal; see the example after Theorem 5.1.

\section{Appendix B.}

Here we shall prove the following result from Calculus which was used in the proof of Theorem 5.1.

Theorem B.1. Let u be a $C^{n}$-function defined on a neighborhood I of the origin and satisfying

$$
u(0)=u^{\prime}(0)=\cdots=u^{(r)}(0)=0
$$

where $r \leq n$. Then there exists a $C^{n-r-1}$-function $v$ on $I$ such that

$$
u(t)=t^{r+1} v(t)
$$

for all $t \in I$.

Applying the following lemma $r+1$ times immediately proves the theorem.

Lemma B.2. Let $u$ be a $C^{k}$-function defined on a neighborhood $I$ of the origin and satisfying $u(0)=0$ where $k \geq 1$. Then there exists a $C^{k-1}$-function $v$ on $I$ such that $u(t)=t v(t)$ for all $t \in I$.

Proof. We have that

$$
u(t)=\int_{0}^{1} \frac{d u(t s)}{d s} d s=\int_{0}^{1} t u^{\prime}(t s) d s=t v(t)
$$

where

$$
v(t):=\int_{0}^{1} u^{\prime}(t s) d s
$$

It is clear that $v$ is continuous. 
Remark. Lemma B.2 can be found on p. 89 in [Ar1] with the redundant assumption that $u^{\prime}(0)=0$.

\section{References}

[Ar1] V. Arnold: Équations différentielles ordinaires. Éditons Mir, Moscou, 1974.

[Ar2] V. Arnold: Remarks on the extatic points of plane curves. In: The Gelfand Mathematical Seminars, 1993-1995, 11-22, Birkhäuser, Boston, 1996.

[Ba] M. Barner: Über die Mindestanzahl stationärer Schmiegebenen bei geschlossenen strengkonvexen Raumkurven. Abh. Math. Sem. Univ. Hamburg 20 (1956), 196-215.

[Bl] W. Blaschke: Vorlesungen über Differentialgeometrie II, Die Grundlehren der Mathematischen Wissenschaften, Band 7, Springer-Verlag, Berlin 1923.

[Bo] R. C. Bose: On the number of circles of curvature perfectly enclosing or perfectly enclosed by a closed convex oval. Math. Z. 35 (1932), 16-24.

[Co] W.A. Coppel: Disconjugacy. Lecture Notes in Math. 220, Springer-Verlag, Berlin etc., 1971.

[GMO] L. Guieu, E. Mourre \& V. Yu. Ovsienko: Theorem on six vertices of a plane curve via Sturm theory. In: The Arnold-Gelfand Mathematical Seminars, pp. 257-266, Birkhäuser, Boston, 1997.

[Ha] O. Haupt: Verallgemeinerung eines Satzes von R. C. Bose über die Anzahl der Schmiegkreise eines Ovals, die vom Oval umschlossen werden oder das Oval umschließen. J. Reine Angew. Math. 239/240 (1969), 339-352.

[HK] O. Haupt and H. Künneth: Geometrische Ordnungen. Die Grundlehren der Mathematischen Wissenschaften, Band 133, Springer-Verlag, Berlin, New York, 1967.

[Hy] T. Hayashi: Some geometrical applications of Fourier series. Rend. Circ. Mat. Palermo 50 (1926), 96-102.

[Ja] S.B. Jackson: Vertices of plane curves. Bull. Amer. Math. Soc. 50 (1944), 564-578.

[Kn] H. Kneser: Neuer Beweis des Vierscheitelsatzes. Christiaan Huygens 2 (1922/23), $315-318$.

[Mu1] S. Mukhopadhyaya: New methods in the geometry of a plane arc, I. Bull. Calcutta Math. Soc. 1 (1909), 31-37. Also in: Collected geometrical papers, vol. I. Calcutta University Press, Calcutta, 1929, 13-20.

[Mu2] S. Mukhopadhyaya: Sur les nouvelles méthodes de géometrie. C. R. Séance Soc. Math. France, année 1933 (1934), 41-45.

[Nö] G. Nöbeling: Über die Anzahl der ordnungsgeometrischen Scheitel von Kurven II. Geometriae Dedicata 31 (1989), 137-149.

[OT] V. Ovsienko \& S. Tabachnikov: Sturm theory, Ghys theorem on zeros of the Schwarzian derivative and flattening of Legendrian curves. Selecta Math. (New Series) 2 (1996), 297-307.

[Ta] S. Tabachnikov: Parametrized plane curves, Minkowski caustics, Minkowski vertices and conservative line fields. Enseign. Math. (2) 43 (1997), 3-26.

[TU1] G. Thorbergsson \& M. Umehara: A unified approach to the four vertex theorems, II. In: Differential and symplectic topology of knots and curves, pp. 229-252, American Math. Soc. Transl. (Series 2) 190, Amer. Math. Soc., Providence, R.I., 1999.

[TU2] G. Thorbergsson \& M. Umehara: Sextactic points on a simple closed curve. Preprint. 
[Um] M. Umehara: A unified approach to the four vertex theorems, I. In: Differential and symplectic topology of knots and curves, pp. 185-228, American Math. Soc. Transl. (Series 2) 190, Amer. Math. Soc., Providence, R.I., 1999.

Gudlaugur Thorbergsson

Mathematisches Institut

Universität zu Köln

Weyertal 86 - 90

50931 Köln

Germany

gthorbergsson@mi.uni-koeln.de
Masaaki Umehara

Department of Mathematics

Graduate School of Science,

Hiroshima University

Higashi-Hiroshima 739-8526

Japan

umehara@math.sci-hiroshima-u.ac.jp 